\title{
Probing the dark matter of a three-loop radiative neutrino mass generation model with the Cherenkov Telescope Array
}

\author{
Talal Ahmed Chowdhury $\odot,{ }^{1,2, *}$ Saquib Hassan $\odot,{ }^{3, \dagger}$ Jahid Hossain $\odot,{ }^{4, \sharp}$ \\ Salah Nasriø ${ }^{5,2,8}$ and Mahmud Ashraf Shamim $\oplus^{6, \pi}$ \\ ${ }^{1}$ Department of Physics, University of Dhaka, P.O. Box 1000, Dhaka, Bangladesh \\ ${ }^{2}$ The Abdus Salam International Centre for Theoretical Physics, Strada Costiera 11, I-34014 Trieste, Italy \\ ${ }^{3}$ Rudolf Peierls Centre for Theoretical Physics, University of Oxford, Oxford OX1 3PU, United Kingdom \\ ${ }^{4}$ Department of Physics and Astronomy, University of Nebraska-Lincoln, \\ Lincoln, Nebraska 68588-0299, USA \\ ${ }^{5}$ Department of Physics, UAE University, P.O. Box 17551, Al-Ain, United Arab Emirates \\ ${ }^{6}$ Department of Physics, DPS STS School Dhaka, P.O. Box 1230, Dhaka, Bangladesh
}

(Received 22 August 2020; accepted 13 January 2021; published 3 February 2021)

\begin{abstract}
We investigate the prospect of detecting the dark matter (DM) candidate in the three-loop radiative neutrino mass generation model extended with large electroweak multiplets of the Standard Model (SM) gauge group, at the future imaging atmospheric Cherenkov telescope known as the Cherenkov Telescope Array (CTA). We find that the addition of such large electroweak multiplets leads to a sizable Sommerfeld enhanced annihilation of the $\mathrm{DM}$ with an $O(\mathrm{TeV})$ mass, into the $\mathrm{SM}$ gauge bosons, which results in continuum- and line-like spectra of very high-energy (VHE) gamma rays, and therefore becomes observable for the CTA. We determine the viable models by setting the upper limit on the $S U(2)_{L}$ isospin of the multiplets from the partial-wave unitarity constraints and the appearance of a low-scale Landau pole in the gauge coupling. Afterwards, by considering the continuum VHE gamma rays produced from the DM annihilation at the Galactic Center, we probe the parameter space of the model using the sensitivity reach of the CTA.
\end{abstract}

DOI: 10.1103/PhysRevD.103.035002

\section{INTRODUCTION}

In recent years, the Imaging Atmospheric Cherenkov Telescopes (IACTs) have not only opened new avenues for ground-based very high-energy gamma-ray astronomy [1-3] but have also offered a testing ground for the dark matter $(\mathrm{DM})$ of the Universe [4,5]. The parameter space of the DM, which has electroweak quantum numbers and mass in the $\mathrm{GeV}-\mathrm{TeV}$ range, has been probed by the currently operating major IACTs: the High Energy Stereoscopic System (H.E.S.S.) [6-10], the Major Atmospheric Gamma Imaging Cherenkov Telescope (MAGIC) [11,12], and the Very Energetic Radiation Imaging Telescope Array System (VERITAS) [13]. The Cherenkov Telescope Array (CTA),

\footnotetext{
*talal@du.ac.bd

saquib.hassan@physics.ox.ac.uk

\#hossain2@ huskers.unl.edu

snasri@uaeu.ac.ae

"mahmud.ashamim84@gmail.com
}

Published by the American Physical Society under the terms of the Creative Commons Attribution 4.0 International license. Further distribution of this work must maintain attribution to the author(s) and the published article's title, journal citation, and DOI. Funded by SCOAP ${ }^{3}$. which is an ongoing international development project for a next-generation IACT, will have the capability to observe gamma rays with energies from $20 \mathrm{GeV}$ to at least $300 \mathrm{TeV}$ over a large area and wide range of view (up to $10^{\circ}$ ) with more than 100 telescopes located in the Northern and Southern Hemispheres. This will allow the CTA to achieve a sensitivity about a factor of 10 better than current instruments such as H.E.S.S., MAGIC, or VERITAS [14-30]. The DM charged under the Standard Model (SM) gauge group and with the mass in the multi-TeV range can produce highly energetic diffuse or monochromatic gamma rays (based on the annihilation channels), which will be within the observational reach of the CTA. Therefore, in this paper, we have explored the detection possibility of the $\mathrm{TeV} \mathrm{DM}$ candidate of the three-loop radiative neutrino mass model, known as the Krauss-Nasri-Trodden model, at the CTA.

The Krauss-Nasri-Trodden (KNT) model [31] is one of the early models of radiative neutrino mass generation (for a comprehensive review, please see Ref. [32]) which ties the origin and smallness of the neutrino mass with the DM of the Universe. The additional beyond-Standard-Model (BSM) fields of the model are two single charged singlet scalars, $S_{1}^{+}, S_{2}^{+}$, and three SM singlet right-handed neutrinos, $N_{R_{i}}, i=1,2,3$, with masses that lie in the GeV-TeV 
range. There is a $Z_{2}$ symmetry, $\left\{S_{2}^{+}, N_{R_{i}}\right\} \rightarrow\left\{-S_{2}^{+},-N_{R_{i}}\right\}$, which not only omits the tree-level Dirac mass term, $\overline{L_{L}} N_{R_{i}} H$, where $L_{L}$ and $H$ are the SM left-handed lepton doublet and Higgs doublet, respectively, but also stabilizes the lightest singlet right-handed neutrino $N_{R_{1}}$ to play the role of DM. Besides this, the KNT model can be generalized [33] by replacing $S_{2}^{+}$with $\boldsymbol{\Phi}$, having integer isospin and hypercharge $Y=1$, and $N_{R_{i}}$ with $\mathbf{F}_{i}$, having integer isospin and $Y=0$ under the SM gauge group, which leaves the three-loop neutrino mass topology invariant. In the generalized KNT model, the lightest neutral fermion component, $F_{1}^{0}$, is the viable DM candidate. Such replacements in the KNT model with large electroweak multiplets have been studied for triplet [34], 5-plet [35], and 7-plet [36] cases. In Ref. [37], it has been shown that for most of the DM mass range from 1 to $50 \mathrm{TeV}$, the Sommerfeld enhanced annihilation cross section to SM gauge bosons in the 5-plet and 7-plet cases are already within reach of H.E.S.S. and the future CTA, because more charged component fields of the fermion multiplet contribute to the enhancement and thus lead to a larger annihilation cross section. Therefore, to probe viable DM candidates in the class of generalized KNT models, we are left with only the minimal model with a singlet fermion and the next-tominimal triplet model where DM is the neutral component of a fermion triplet.

The DM of the minimal KNT model, being a singlet under the SM gauge group, has different DM parameter space [38] than the triplet or 5-plet and 7-plet models, which have dominant gauge interactions controlling the viable DM parameter space. For this reason, in our CTA sensitivity study, we have addressed the triplet DM, because it shares the same region of parameter space as the 5-plet and 7-plet, and we have left the singlet case for a separate analysis.

The article is organized as follows: In Sec. II, we present the model and calculate the possible upper bound on the $S U(2)_{L}$ isospin of the electroweak multiplets in the generalized KNT model. In Sec. III, we describe the Sommerfeld enhanced DM annihilation cross section and the gamma-ray flux originating from the DM annihilation in the triplet KNT model. Section IV delineates the working principle of the CTA, its instrument response functions, and possible sources of background, and lastly gives the observation region we consider in our study. In Sec. V, we outline our methods to determine the expected gamma-ray counts from the DM signal and backgrounds, and we use the likelihood analysis to set the upper limit on the DM annihilation cross section in the KNT model. Finally, we conclude in Sec. VI. In Appendix A, we present the detailed calculations relevant for our partial-wave unitarity constraints.

\section{THE MODEL}

The BSM fields in the generalized KNT model, charged under SM gauge group $S U(3)_{c} \times S U(2)_{L} \times U(1)_{Y}$, are

$$
\text { complex scalars: } S_{1}^{+} \sim(0,0,1), \quad \boldsymbol{\Phi} \sim\left(0, j_{\phi}, 1\right),
$$

and dreal fermions: $\mathbf{F}_{1,2,3} \sim\left(0, j_{F}, 0\right)$,

where $j_{\phi}$ and $j_{F}$ are integer isospins of $S U(2)_{L}$. For example, the triplet model would contain $\Phi \sim(0,1,1)$ and $\mathbf{F}_{1,2,3} \sim(0,1,0)$.

The generalized KNT Lagrangian that has the additional $Z_{2}$ symmetry is given by

$$
\begin{aligned}
\mathcal{L} \supset & \mathcal{L}_{\mathrm{SM}}+\left\{f_{\alpha \beta} \overline{L_{\alpha}^{c}} \cdot L_{\beta} S_{1}^{+}+g_{i \alpha} \overline{\mathbf{F}_{i}} \cdot \boldsymbol{\Phi} . e_{\alpha_{R}}+\text { H.c. }\right\} \\
& -\frac{1}{2} \overline{\mathbf{F}_{i}^{c}} M_{F_{i j}} \mathbf{F}_{j}-V\left(H, \boldsymbol{\Phi}, S_{1}\right)+\text { H.c. }
\end{aligned}
$$

where $c$ denotes the charge conjugation, and the dot sign, in shorthand, refers to appropriate $S U(2)$ contractions. Also, $L_{\alpha}$ and $e_{R_{\alpha}}$ are the left-handed (LH) lepton doublet and right-handed (RH) charged leptons, respectively, and the greek $\alpha$ stands for the flavor index, i.e. $\alpha=e, \mu, \tau$. Moreover, $f_{\alpha \beta}$ and $g_{i \alpha}$ (where $i$ is the generation index of the fermionic multiplets, $i=1,2,3$ ) are the Yukawa couplings appearing in Eq. (2), which can be considered as the components of a complex antisymmetric matrix $F$ and a general complex matrix $G$, respectively. $H$ is the SM Higgs doublet. Finally, $V\left(H, \boldsymbol{\Phi}, S_{1}\right)$ denotes the scalar potential.

We have seen that the generalized KNT model contains $S U(2)_{L}$ fermionic and scalar multiplets of integer isospins. In the case of the minimal KNT model [31], the Yukawa term of Eq. (2), $g_{i \alpha} \overline{\mathbf{F}_{i}} \cdot \boldsymbol{\Phi} \cdot e_{\alpha_{R}}$, boils down to the term containing three real Majorana fermions, $\mathbf{F}_{i} \equiv N_{i}$ which are SM singlet, and a single-charged $S U(2)_{L}$ singlet scalar, $\Phi \equiv S_{2}^{+}$. Therefore, in the generalized KNT model, one would require three real $S U(2)_{L}$ fermionic multiplets $\mathbf{F}_{i}$ to embed the minimal KNT model's fermions as one of the component fields in those multiplets. This requires $\mathbf{F}_{i}$ to have integer isospin and zero hypercharge. Now, the SM invariance of the Yukawa term forces the $S U(2)_{L}$ scalar multiplet $\Phi$ to have integer isospin equal to that of the fermionic multiplets-i.e., $j_{\phi}=j_{F}$-and hypercharge $Y=1$. As we can see, the single-charged scalar of the minimal KNT model is now a component field of the complex scalar multiplet.

The mass splittings in fermionic component fields are zero at tree level and only receive $O(100 \mathrm{MeV})$ splittings due to the radiative correction after the electroweak symmetry breaking [39-41]. On the other hand, the mass splittings among component fields of the scalar multiplet $\boldsymbol{\Phi}$ are controlled by the $\lambda_{H \phi 2}\left(\boldsymbol{\Phi}^{\dagger} . H\right) .\left(H^{\dagger} . \boldsymbol{\Phi}\right)$ term of the scalar potential after electroweak symmetry breaking. The splittings $\Delta m$ allowed by the electroweak precision observables only lead to $\Delta m_{i j}^{2} / M_{0}^{2} \sim 10^{-3}$ if the invariant

\footnotetext{
${ }^{1}$ We consider the electric charge to be $Q=T^{3}+Y$, where $T^{3}$ and $Y$ are the diagonal generators of $S U(2)_{L}$ and $U(1)_{Y}$, respectively.
} 
mass of the scalar multiplet is $M_{0}=10 \mathrm{TeV}$, and for $M_{0}>10 \mathrm{TeV}$, the ratio becomes even smaller, as shown in Ref. [42]. Therefore, the component fields of both scalar and fermion multiplets are almost degenerate at the $\mathrm{TeV}$ scale. Consequently, such near degeneracy of fermion component fields does not diminish the Sommerfeld enhancement of DM annihilation.

As we have seen, the large isospin multiplets of the $S U(2)_{L}$ are allowed in the generalized KNT model, so one could ask for the upper bound on the electroweak quantum numbers for such large multiplets. In the following Secs. II A and II B, we have used the arguments from the partial-wave unitarity and appearance of the low-scale Landau pole to set an upper limit on the isospin in the generalized KNT model.

\section{A. Partial-wave unitarity constraints}

To set the partial-wave unitarity constraints on the electroweak quantum numbers of large multiplets in the KNT model, we have looked into the tree-level scattering of the component fields of the fermion multiplets into the SM gauge bosons, $F F \rightarrow V V$, i.e., into $W W, Z Z, \gamma \gamma, \gamma Z$, as shown in Fig. 1. Similar analysis with SM gauge bosons as the final states was done in Ref. [43]. Moreover, the upper bound on the isospin for the scalar multiplet using the same final states was addressed by Ref. [44].

Let us consider the $2 \rightarrow 2$ scattering amplitude in momentum space with initial and final two-particle states $|i\rangle$ and $|f\rangle$, respectively, as

$$
\langle f|T| i\rangle=(2 \pi)^{4} \delta^{4}\left(P_{f}-P_{i}\right) \mathcal{T}_{f i}(\sqrt{s}, \cos \theta),
$$

where $s$ is the center-of-mass energy. Here $T$ captures the interaction part of the $S$ matrix, $S=1+i T$. From Eq. (3), by using the Jacob-Wick expansion [45-47], the corresponding partial-wave amplitude of total angular momentum $J$ is

$$
\begin{aligned}
a_{f i}^{J}= & \frac{\beta^{1 / 4}\left(s, m_{i_{1}}^{2}, m_{i_{2}}^{2}\right) \beta^{1 / 4}\left(s, m_{f_{1}}^{2}, m_{f_{2}}^{2}\right)}{32 \pi s} \\
& \times \int_{-1}^{1} d(\cos \theta) d_{\mu_{i} \mu_{f}}^{J}(\theta) \mathcal{T}_{f i}(\sqrt{s}, \cos \theta),
\end{aligned}
$$

where $d_{\mu_{i} \mu_{f}}^{J}$ is the $J$ th Wigner $d$ function, with $\mu_{i}=\lambda_{i_{1}}-\lambda_{i_{2}}$ and $\mu_{f}=\lambda_{f_{1}}-\lambda_{f_{2}}$ defined in terms of the helicities of the initial $\left(\lambda_{i_{1}}, \lambda_{i_{2}}\right)$ and final $\left(\lambda_{f_{1}}, \lambda_{f_{2}}\right)$ states. In addition, $\beta(x, y, z)=x^{2}+y^{2}+z^{2}-2 x y-2 y z-2 z x$ is the Kallen function. Moreover, a factor of $1 / \sqrt{2}$ should be multiplied at the right-hand side of Eq. (4) for any identical pairs of particles in either the initial or final states.

The unitarity condition on the $S$ matrix, $S^{\dagger} S=1$, implies that

$$
\frac{1}{2 i}\left(a_{f i}^{J}-a_{i f}^{J *}\right) \geq \sum_{n} a_{f n}^{J *} a_{n i}^{J}
$$

where the inequality arises because the sum over $n$ is restricted to two-particle states only. If we consider elastic scattering, for which $f=i$, we have from Eq. (5)

$$
\operatorname{Im} a_{i i}^{J} \geq\left|a_{i i}^{J}\right|^{2}+\sum_{k \neq i}\left|a_{k i}^{J \text { inel }}\right|^{2}
$$

where the sum over $k \neq i$ is taken over all of the possible two-particle inelastic channels. By writing $\left|a^{J}\right|^{2}=$ $\left(\operatorname{Re} a^{J}\right)^{2}+\left(\operatorname{Im} a^{J}\right)^{2}$, we have

$$
\operatorname{Im} a_{i i}^{J}\left(1-\operatorname{Im} a_{i i}^{J}\right) \geq\left(\operatorname{Re} a_{i i}^{J}\right)^{2}+\sum_{k \neq i}\left|a_{k i}^{J \text { inel }}\right|^{2} .
$$

Now that $a_{i i}^{J}$ lies on the unitary circle, the left-hand side of Eq. (7) is bounded by $1 / 4$, and we can have in the absence of any inelastic channel

$$
\operatorname{Re} a_{i i}^{J} \leq \frac{1}{2}
$$

Therefore, the maximal possible bound one can set on the real part of an inelastic channel $k$ is

$$
\operatorname{Re} a_{k i}^{J \text { inel }} \leq \frac{1}{2}
$$

In practice, one can consider the full transition matrix at tree level that connects all possible two-particle states and impose the bound Eqs. (8) or (9) on the largest eigenvalue of the matrix, $\left|\operatorname{Re} a_{f i}^{J}\right|$.

Now, the tree-level scattering of the component fields of the fermion multiplets into SM gauge bosons can be classified into coupled channels based on the electric

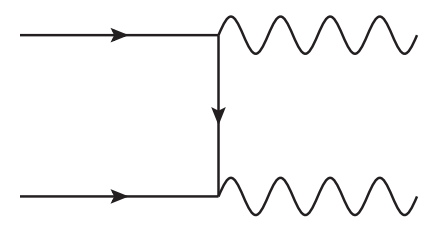

(a)

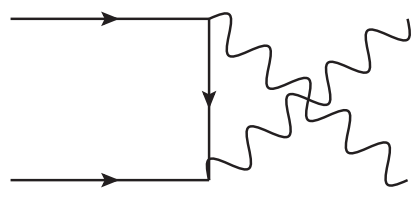

(b)



(c)

FIG. 1. Partial-wave unitarity constraints on scattering processes $F F \rightarrow V V$. 

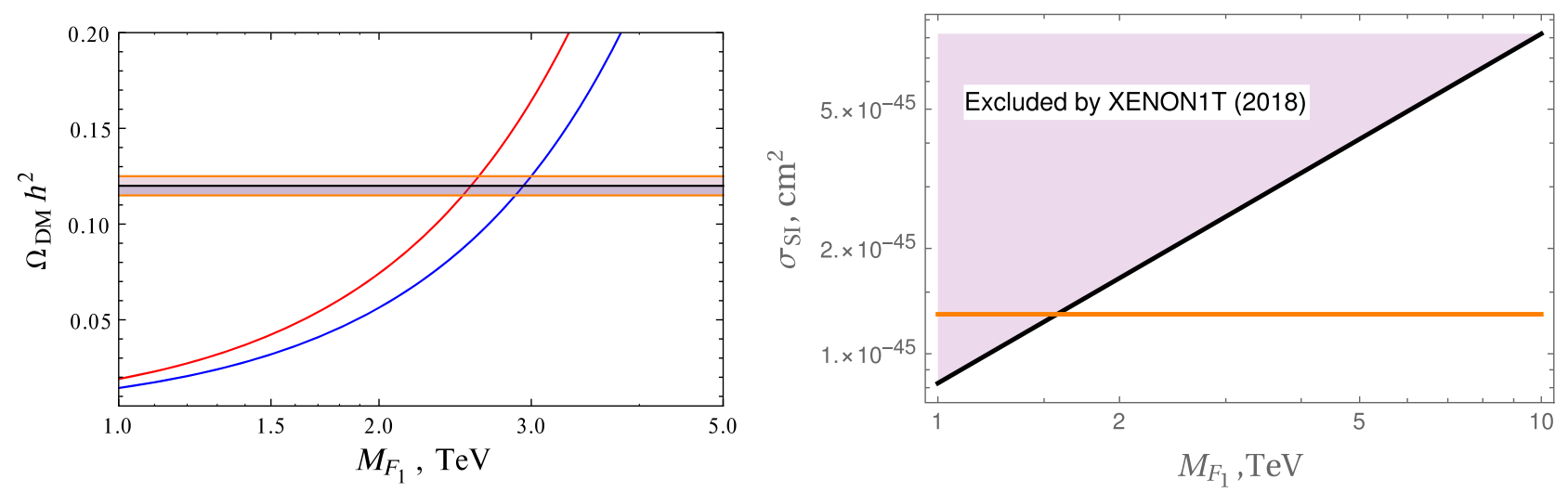

FIG. 2. Left: the DM relic densities $\Omega h^{2}$ of a triplet without SE (red) and with SE (blue). The horizontal band represents the $5 \sigma$ band with the central value $\Omega h^{2}=0.12$ measured by Planck. Right: the spin-independent cross section of triplet DM, $F_{1}^{0}$, and nucleon interaction (orange horizontal line). The shaded region is excluded by XENON1T (2018) data [53].

charge conservation. That leads to three charge sectors for $2 \rightarrow 2$ channels:

$$
\begin{aligned}
& Q_{\mathrm{tot}}=0: F_{i}^{(Q)} F_{i}^{(-Q)} \rightarrow W^{+} W^{-}, Z Z, \gamma \gamma, \gamma Z, \\
& Q_{\mathrm{tot}}=1: F_{i}^{(Q+1)} F_{i}^{(-Q)} \rightarrow W^{+} Z, W^{+} \gamma, \\
& Q_{\mathrm{tot}}=2: F_{i}^{(Q+2)} F_{i}^{(-Q)} \rightarrow W^{+} W^{+} .
\end{aligned}
$$

In practice, one can determine the largest eigenvalue associated with each coupled-channel transition matrix with $Q_{\mathrm{tot}}=0,1,2$ and impose the bound in Eq. (9), but, as the coupled-channel transition matrix for $Q_{\mathrm{tot}}=0$ constrains more channels into SM gauge bosons compared to the charged sectors $Q_{\mathrm{tot}}=1,2$, we consider it to determine the possible upper limit on the $S U(2)$ isospin. Finally, for the $Q_{\text {tot }}=0$ sector, the bound given in Eq. (9) implies that the isospin of the fermionic multiplet in the KNT model (see Appendix A for detailed calculation) has to be

$$
\begin{array}{ll}
j_{F} \leq 8 & \text { (one generation) and } \\
j_{F} \leq 7 & \text { (three generations). }
\end{array}
$$

One could also include the elastic channels $F_{i} \bar{F}_{i} \rightarrow F_{i} \bar{F}_{i}$ in the coupled-channel matrix. But the helicity amplitude, with helicity transition $0 \rightarrow 0$, in the $t$ channel via photon exchange has a singularity at $\theta=0$. Such presence of the Coloumb singularity in the elastic channel renders the partial-wave unitarity analysis somewhat ineffective. Therefore, to put a more concrete bound on the electroweak quantum numbers, one has to look into the appearance of the low-scale Landau pole in the SM gauge couplings in the presence of large electroweak multiplets.

\section{B. Appearence of the Landau pole in gauge coupling}

The beta functions of the SM gauge couplings are modified when the larger electroweak fermionic or scalar multiplets are added to their matter content. If the isospin and the hypercharge of the $i$ th fermionic and $j$ th scalar multiplets are $\left(j_{F_{i}}, Y_{F_{i}}\right)$ and $\left(j_{S_{j}}, Y_{S_{j}}\right)$, respectively, then the one-loop beta functions for the $S U(2)_{L}$ and $U(1)_{Y}$ gauge couplings $g$ and $g_{Y}$, respectively, become

$$
\beta_{g}=\frac{g^{3}}{16 \pi^{2}}\left(-\frac{19}{6}+\frac{4}{3} \sum_{i} \kappa_{F_{i}} T\left(F_{i}\right)+\frac{1}{3} \sum_{j} \eta_{S_{j}} T\left(S_{j}\right)\right)
$$

$$
\beta_{g_{Y}}=\frac{g_{Y}^{3}}{16 \pi^{2}}\left(\frac{41}{6}+\frac{4}{3} \sum_{i} \kappa_{F_{i}} D\left(F_{i}\right) Y_{F_{i}}^{2}+\frac{1}{3} \sum_{j} \eta_{S_{j}} D\left(S_{j}\right) Y_{S_{j}}^{2}\right)
$$

Here, $-\frac{19}{6}$ and $\frac{41}{6}$ within the parentheses are the SM contributions to the $g$ and $g_{Y}$ beta functions, respectively. $\kappa_{F_{i}}=1, \frac{1}{2}$ if the $i$ th fermionic multiplet contains Dirac or Weyl fermions; $\eta_{S_{j}}=1, \frac{1}{2}$ if the $j$ th scalar multiplet contains complex and real scalars, respectively. In addition, the Dynkin index $T(R)$ of the fermionic or scalar multiplet $R$ with isospin $j$ is given by $T(R)=j(j+1)(2 j+1) / 3$, and its corresponding dimension ${ }^{2}$ is given as $D(R)=2 j+1$. As the last two terms of Eq. (11) give a positive contribution to the beta function of $g$, for large enough isospin,

\footnotetext{
${ }^{2}$ The Dynkin index $T(R)$ for representation $R$ is defined, for example, in Refs. [88,89], as $C_{2}(R) D(R)=T(R) D$ (Adj), where $D(R)$ and $D(\operatorname{Adj})$ are the dimensions of the representation $R$ and adjoint representation, respectively, and $C_{2}(R)$ is the Casimir invariant associated with representation $R$ given by $C_{2}(R) \mathbb{I}=$ $\sum_{a} T_{R}^{a} T_{R}^{a}$, where $T_{R}^{a}$ are the generators in representation $R$, and $\mathbb{I}$ is an identity matrix of order $D(R)$. For $S U(2)$ representation $R$ with isospin $j$, the dimension is $D(R)=2 j+1$, the Casimir invariant is $C_{2}(R)=j(j+1)$, and the expression for $T(R)$ follows.
} 
TABLE I. Appearance of the Landau pole for $S U(2)_{L}$ gauge coupling with the isospin $j$ in the generalized KNT model. Here, $M_{X}$ is the mass scale where the additional electroweak multiplets are integrated in.

\begin{tabular}{lcccr}
\hline \hline \multicolumn{5}{c}{ Mass scale, $M_{X}$} \\
\hline$S U(2)$ Isospin, $j$ & $M_{Z}=91.1876 \mathrm{GeV}$ & $10^{3} \mathrm{GeV}$ & $10^{4} \mathrm{GeV}$ & $10^{5} \mathrm{GeV}$ \\
\hline 1 & $4.6 \times 10^{16} \mathrm{GeV}$ & $2 \times 10^{18} \mathrm{GeV}$ & $\gg M_{p}$ & $\gg M_{p}$ \\
2 & $9.4 \times 10^{3} \mathrm{GeV}$ & $1.2 \times 10^{5} \mathrm{GeV}$ & $1.5 \times 10^{6} \mathrm{GeV}$ & $1.7 \times 10^{7} \mathrm{GeV}$ \\
3 & $440 \mathrm{GeV}$ & $5.1 \times 10^{3} \mathrm{GeV}$ & $5.4 \times 10^{4} \mathrm{GeV}$ & $5.8 \times 10^{5} \mathrm{GeV}$ \\
\hline \hline
\end{tabular}

they can overcome the negative SM contribution and make it positively large. As a consequence, such a large positive beta function will lead to the appearance of the Landau pole for the $S U(2)_{L}$ gauge coupling at a scale much lower than the Planck scale. As we have not observed nonperturbative weak interaction at energy scales a few orders above the electroweak scale, the appearance of such a low-scale Landau pole puts a severe constraint on the size of the electroweak multiplet.

For the KNT model, the additional three fermionic multiplets with $S U(2)$ isospin $j$ and hypercharge $Y=0$, and one scalar multiplet with isospin $j$ and hypercharge $Y=1$, will contribute to Eq. (11), and depending on the mass scale $M_{X}$, where the multiplets are integrated in during the renormalization group running, we tabulate the Landau pole for $g$ in Table I (see Appendix B for details).

The additional BSM fields of the KNT model which contribute to the neutrino mass generation radiatively at three loops can have masses ranging from the electroweak to the $\mathrm{TeV}$ scale, and here they are denoted by a common mass $M_{X}$ for simplicity. Therefore, if we consider that the masses of these KNT fields are not too heavy compared to the EW scale-i.e., $M_{X} \simeq M_{Z}-$ we can see from Table I that the Landau pole of the $S U(2)$ coupling $g$ appears at $O(\mathrm{TeV})$ for the 5-plet $(j=2)$ and at $440 \mathrm{GeV}$ for the 7-plet $(j=3)$. In contrast, if masses of the KNT fields are at the $O(\mathrm{TeV}-100 \mathrm{TeV})$ range-i.e., $M_{X} \gg M_{Z}$-and if we consider their contributions to RG running of the $S U(2)$ coupling below $M_{X}$ to be negligible, the Landau poles for the 5-plet and 7-plet can be pushed to higher energies by integrating in the KNT multiplets at higher $M_{X}$, but not as high as the Planck scale, as we can see for the triplet case $(j=1)$. As the presence of KNT multiplets with $j \geq 1$ eventually leads to the appearance of the Landau pole for $S U(2)$ gauge coupling $g$ at high energy, we want to know how the KNT mass scale $M_{X}$ or integrating in these BSM multiplets at higher energies in our simplified RG running of $g$ would affect the scale of its corresponding Landau pole. But the KNT model's full parameter space contains masses of these additional BSM fields ranging from the EW to the multi-TeV scale; therefore, from a conservative point of view we can consider the $M_{X}$ to be close to the EW scale. ${ }^{3}$ Since we have not seen the signature of new physics in the electroweak scale at energies accessible by the LHC, the appearance of the low-scale Landau pole of the SM gauge coupling for electroweak multiplets with $j \geq 2$ has made the singlet $(j=0)$ and the triplet a viable BSM addition for the KNT model.

One could improve the analysis of the size of the electroweak multiplet and the Landau pole by using the two-loop beta functions of the gauge couplings as done in Refs. [48,49]. However, we refrain from using the two-loop beta functions, because first, we do not know the UV completion of the KNT model and second, the KNT Yukawa coupling $g_{i \alpha}$ enters into the two-loop beta functions of the gauge couplings, and eventually the two-loop Landau pole will also depend on these couplings. For this reason, the size of the electroweak multiplets will not be connected to the appearance of the low-scale Landau pole in a straightforward way as in the one-loop case. However, even if we consider the UV completion of the model with large electroweak multiplets in a Grand Unified Theory (GUT) setup, it would require either enormous representations of the minimal GUT like $S O(10)$ or a much bigger GUT group, where the model can be embedded in its

\footnotetext{
${ }^{3}$ In the case of the KNT model, the radiatively generated neutrino mass is directly proportional to the charged lepton masses and the loop function, $\mathcal{F}\left(M_{F_{i}}^{2} / M_{\Phi}^{2}, M_{S_{1}}^{2} / M_{\Phi}^{2}\right)$, which becomes smaller when $M_{F_{i}}^{2} / M_{\phi}^{2} \rightarrow 0$ and $\infty$, and inversely proportional to $M_{\Phi}$. Here $M_{F_{i}}$ and $M_{\Phi}$ are the common masses of the fermion and scalar multiplets, respectively [as the mass splittings among the component fields are of $O(100 \mathrm{MeV})]$, and $M_{S_{1}}$ is the mass of the singly charged scalar singlet. Therefore, for a fixed $M_{\phi}$ around $\mathrm{TeV}$, the fermion mass $M_{F_{i}} \ll M_{Z}$ or $M_{F_{i}} \gg O(\mathrm{TeV})$ would lead to the smaller neutrino masses, which would have been incompatible with the experimental bound. So, the low-energy neutrino constraints allow the masses of the KNT fields to range from close to the EW scale to the $O(\mathrm{TeV})$ range. On the other hand, allowing for the neutral component of the lightest fermion multiplet to satisfy the DM relic density constraint via the thermal freeze-out mechanism sets the masses of the fermion and scalar multiplets in the $\mathrm{TeV}$ range. But one can invoke nonstandard DM production mechanisms in the KNT model, which can again relax this TeV mass-range requirement. For this reason, we consider the mass scale $M_{X}$ from close to the EW scale to $O(\mathrm{TeV})$ scales.
} 
fundamental or adjoint representations, both of which are not theoretically appealing.

\section{GAMMA-RAY FLUX FROM DARK MATTER ANNIHILATION}

\section{A. Sommerfeld enhanced DM annihilation}

In the triplet KNT model, the lightest neutral component of the fermion triplet, $F_{1}^{0}$, is the viable DM candidate. The Sommerfeld enhancement of the DM annihilation to SM gauge bosons takes place when the DM is nonrelativistic, $v_{\mathrm{DM}} \ll c$, and the SM gauge bosons follow $m_{W, Z} \ll m_{\mathrm{DM}}$. In this limit, the exchange of $W$ and $Z$ bosons between triplet component fields will lead to Yukawa potentials, and $\gamma$ exchange will lead to a Coulomb potential, which in turn significantly modifies the wave function of the incoming DM states and enhances the annihilation cross sections. The calculation of the Sommerfeld enhanced DM annihilation cross section is a well-studied subject, so here we follow the prescriptions given in Ref. [50]. Nevertheless, we briefly review them to set up our notation.

As the Sommerfeld enhancement is considered for $2 \rightarrow 2$ processes, we first define two-particle states which consist of incoming component fields of fermion triplets. For the DM (co)annihilation channels where the final states consist of $W^{ \pm}, Z$, and $\gamma$ bosons, the only relevant twoparticle states are $C P$-even states with total electric charges $Q=0, \pm 1, \pm 2$. In the case of DM annihilation in the Galactic halo at present times, only two-particle states with $Q=0$ are applicable.

In general, if the three fermionic triplets of the KNT model are almost mass degenerate, $M_{F_{1}} \sim M_{F_{2}} \sim M_{F_{3}}$, the two-particle states will contain component fields not only from one multiplet but also from different ones. But, as the hierarchical mass spectrum of $\mathbf{F}_{i}$ is consistent with the best-fit experimental values of the neutrino mixing angles and mass-squared differences [51], it allows us to have $M_{F_{1}} \ll M_{F_{2,3}}$ and allows $M_{F_{1}}$ to be in $O(\mathrm{TeV})$. It enables us to define a two-particle state vector made out of component fields of $\mathbf{F}_{1}$ as follows:

$$
\begin{gathered}
Q=0:|\Psi\rangle=\left(F_{1}^{0} F_{1}^{0}, F_{1}^{ \pm} F_{1}^{\mp}\right)^{T}, \\
Q= \pm 1:|\Psi\rangle=\left|F_{1}^{0} F_{1}^{ \pm}\right\rangle, \\
Q= \pm 2:|\Psi\rangle=\left|F_{1}^{ \pm} F_{1}^{ \pm}\right\rangle .
\end{gathered}
$$

The modification of the wave function which generates the Sommerfeld enhancement is determined by solving the radial Schrodinger equation with effective potential

$$
\begin{aligned}
& \frac{d^{2} \Psi_{j j^{\prime}, i i^{\prime}}}{d r^{2}}+\left[\left(\left(M_{F_{1}} v\right)^{2}-\frac{l(l+1)}{r^{2}}\right) \delta_{j j^{\prime}, k k^{\prime}}\right. \\
& \left.-M_{F_{1}} V_{j j^{\prime}, k k^{\prime}}\right] \Psi_{k k^{\prime}, i i^{\prime}}=0,
\end{aligned}
$$

where $r$ is the magnitude of the relative distance between two component fields in their center-of-mass frame, and the kinetic energy of the incoming DM states-i.e., $\left|i i^{\prime}=F_{1}^{0} F_{1}^{0}\right\rangle$-is $E=M_{F_{1}} v^{2}$, The wave function $\Psi_{j j^{\prime}, i i^{\prime}}$ gives the transition amplitude from $\left|i i^{\prime}\right\rangle$ states to $\left|j j^{\prime}\right\rangle$ states in the presence of effective potential, $V$. The double indices $i i^{\prime}, j j^{\prime}$, and $k k^{\prime}$ run over the states of the two-particle state vector defined in Eq. (13).

We primarily focus on the $S$-wave annihilation, so we set $l=0$ and have

$$
\begin{aligned}
& \frac{d^{2} \Psi_{j j^{\prime}, i i^{\prime}}}{d r^{2}}+\left[k_{j j^{\prime}}^{2} \delta_{j j^{\prime}, k k^{\prime}}+M_{F_{1}}\left(\frac{f_{j j^{\prime}, k k^{\prime}} \alpha_{a} e^{-n_{a} m_{W} r}}{r}\right.\right. \\
& \left.\left.+\frac{Q_{k k^{\prime}}^{2} \alpha_{\mathrm{em}}}{r} \delta_{j j^{\prime}, k k^{\prime}}\right)\right] \Psi_{k k^{\prime}, i i^{\prime}}=0 .
\end{aligned}
$$

Here, $\quad k_{j j^{\prime}}^{2}=M_{F_{1}}\left(M_{F_{1}} v^{2}-d_{j j^{\prime}}\right)$ is the momentum associated with the two-particle state $\left|j j^{\prime}\right\rangle$, and $d_{j j^{\prime}}=$ $m_{j}+m_{j^{\prime}}-2 M_{F_{1}}$ denotes the mass differences between $\mathrm{DM}$ and other states of the multiplet. $Q_{k k^{\prime}}$ is the electric charge associated with state $\left|k k^{\prime}\right\rangle$. Also, $\alpha_{W}=\alpha$ and $n_{W}=1$ for $W$-boson exchange, and $\alpha_{Z}=\alpha / \cos ^{2} \theta_{W}$ and $n_{Z}=1 / \cos \theta_{W}$ for Z-boson exchange. Finally, $f_{j j^{\prime}, k k^{\prime}}$ is the group theoretical factor associated with $S U(2)$.

Now, by using dimensionless variables defined as $x=\alpha m_{F_{1}} r, \epsilon_{\phi}=\left(m_{W} / m_{F_{1}}\right) / \alpha, \epsilon_{v}=(v / c) / \alpha$, and $\epsilon_{d_{i i^{\prime}}}=$ $\sqrt{d_{i i^{\prime}} / m_{F_{1}}} / \alpha$, we rewrite the coupled radial Schrodinger equations as

$$
\begin{gathered}
\frac{d^{2} \Psi_{j j^{\prime}, i i^{\prime}}}{d x^{2}}+\left[\hat{k}_{j j^{\prime}}^{2} \delta_{j j^{\prime}, k k^{\prime}}+\frac{f_{j j^{\prime}, k k^{\prime}} n_{a}^{2} e^{-n_{a} \epsilon_{\phi} x}}{x}\right. \\
\left.+\frac{Q_{k k^{\prime}}^{2} \sin ^{2} \theta_{W}}{x} \delta_{j j^{\prime}, k k^{\prime}}\right] \Psi_{k k^{\prime}, i i^{\prime}}=0,
\end{gathered}
$$

where the dimensionless momentum $\hat{k}_{j j^{\prime}}^{2}=\epsilon_{v}^{2}-\epsilon_{d_{j j^{\prime}}}^{2}$.

At large $x, \Psi_{j j^{\prime}, i i^{\prime}}$ behaves as $\Psi_{j j^{\prime}, i i^{\prime}} \sim T_{j j^{\prime}, i i^{\prime}} e^{i \hat{k}_{j j^{\prime}} x}$, where $T_{j j^{\prime}, i i^{\prime}}$ is the transition amplitude provided the effective potential is dominated by Yukawa potential. Now, if the annihilation matrix for final state $f$ is given by $\Gamma_{j j^{\prime}, i i^{\prime}}^{(f)}$, the annihilation cross section is

$$
\sigma_{F_{1}^{0} F_{1}^{0} \rightarrow f}=c\left(T^{\dagger} \cdot \Gamma^{(f)} \cdot T\right)_{F_{1}^{0} F_{1}^{0}, F_{1}^{0} F_{1}^{0}},
$$

where $c=2$ for the $\left|F_{1}^{0} F_{1}^{0}\right\rangle$ state, as it consists of identical fields. The Sommerfeld enhancement factor is then given by $S_{F_{1}^{0} F_{1}^{0} \rightarrow f}=\sigma_{F_{1}^{0} F_{1}^{0} \rightarrow f} / \sigma_{F_{1}^{0} F_{1}^{0} \rightarrow f}^{0}$, where $\sigma^{0}$ is the tree-level annihilation cross section.

\section{B. Dark matter constraints and parameter space}

The relic density of DM in the Universe measured by the Planck Collaboration as $\Omega_{\mathrm{DM}} h^{2}=0.120 \pm 0.001$ 
(68\% C. L.) [52] sets an important constraint for KNT triplet DM. In the case of $F_{1}^{0}$, the standard thermal freezeout scenario involves the SM gauge interactions and the KNT Yukawa interactions given by $g_{i \alpha}$ terms in Eq. (2).

The DM (co)annihilation into the SM final states, controlled by the SM gauge interactions, take place via $S$-wave and $P$-wave channels. Moreover, the $S$-wave DM (co)annihilation cross sections receive non-negligible Sommerfeld enhancement. In contrast, the DM annihilation into charged leptons that involves the KNT Yukawa coupling $g_{i \alpha}$ has $S$-wave and $P$-wave channels. However, both of them are suppressed, by the light charged lepton mass and the velocity of the DM, respectively. Because of the large multiplicity and unsuppressed Sommerfeld enhanced $S$-wave contribution, the DM (co)annihilation controlled by gauge interaction is more dominant compared to that into charged leptons involving the KNT Yukawa coupling. Therefore, essentially the gauge interaction determines the relic density of the triplet KNT DM in the thermal freeze-out, which is calculated using nonrelativistic approximation described in Ref. [37] (and references therein).

Another constraint comes from the DM direct detection. The spin-independent cross section for the Majarana DM contained in the electroweak multiplet of integer isospin $j$ does not depend on the DM mass, and is given by [39]

$$
\sigma_{\mathrm{SI}}=j^{2}(j+1)^{2} \frac{\pi \alpha^{2} M_{\mathrm{Nucl}}^{4} f^{2}}{4 m_{W}^{2}}\left(\frac{1}{m_{W}^{2}}+\frac{1}{m_{h}^{2}}\right),
$$

where $M_{\text {Nucl }}$ is the mass of the target nucleus, $f$ parametrizes the nucleon matrix element as $\left\langle n\left|\sum_{q} m_{q} \bar{q} q\right| n\right\rangle=$ $f m_{n} \bar{n} n$, and from the lattice result, $f=0.347131$ [54]. On the other hand, the spin-dependent cross section is suppressed by the mass of the DM which is of the $O(\mathrm{TeV})$.

Therefore, we can see that the constraint from the DM relic density sets the mass of the DM in the triplet KNT model as $2.55 \mathrm{TeV}$ (without SE) and $2.94 \mathrm{TeV}$ (with SE), if the standard thermal freeze-out mechanism is considered. But, as pointed out in Ref. [42], the nonthermal decay of the scalar $\phi^{+}$can produce the DM $F_{1}$ within the KNT model, although some fine-tuning in the mass splittings and couplings is needed to get the correct relic density for a wider range of DM masses. However, the fine-tuning can be avoided if the KNT model is coupled to an extended dark sector which would assist the nonthermal production of the DM. For this reason, we probe the DM in the KNT model with mass up to $100 \mathrm{TeV}$ that already includes the mass set by the relic density constraint for the thermal freeze-out scenario in our CTA sensitivity study.

\section{Gamma-ray flux from DM annihilation}

The gamma rays from the DM annihilation consist of the prompt gamma rays and the secondary gamma rays. The prompt gamma rays are produced directly from the DM annihilation, or the decays of the SM final states originated in the annihilation. On the other hand, the secondary gamma rays come from the inverse Compton scattering (ICS) of $e^{ \pm}$produced in DM annihilation, again directly or from decays of the SM final states, with the ambient photon background-i.e., mainly coming from cosmic microwave background photons, dust rescattered light, and starlight. As our analysis focuses on the diffuse prompt gamma-ray flux coming from the Sommerfeld enhanced DM annihilation into gauge bosons $\left(W^{+} W^{-}\right)$with energy in the $\mathrm{TeV}$ range, we neglect the effect of secondary gamma-ray emission from DM annihilation for simplification.

The differential prompt gamma-ray flux from the DM annihilation for a given DM mass, $m_{F_{1}}$, in the generalized KNT model is

$$
\frac{d \Phi}{d E_{\gamma}}=\frac{\langle\sigma v\rangle}{8 \pi m_{F_{1}}^{2}} \sum_{f} B_{f} \frac{d N_{f}}{d E_{\gamma}} \int_{\Delta \Omega} \int_{\mathrm{LOS}} \rho_{\mathrm{DM}}^{2}(r) d s d \Omega,
$$

where $\langle\sigma v\rangle$ is the corresponding velocity-averaged annihilation cross section for $m_{F_{1}}, d N_{f} / d E_{\gamma}$ is the gammaray spectrum per annihilation for the annihilation channel, $F_{1}^{0} F_{1}^{0} \rightarrow f$, and $B_{f}$ is the corresponding branching ratio. The integration over the solid angle $\Delta \Omega$ and line of sight (LOS) $s$ of the squared DM mass density $\rho_{\mathrm{DM}}$ is called the astrophysical $J$ factor. Here, $r$ is the distance between the Galactic Center (GC) and the point in space characterized by Galactic coordinates $(b, l)$, and it is given as $r(s, \theta)=\left(r_{\odot}^{2}+s^{2}-2 r_{\odot} s \cos \theta\right)^{1 / 2}$, where $r_{\odot}$ is the distance between the Earth and Galactic Center, and $\theta$ is the angle between the line of sight and the axis connecting the Earth and the GC, as shown in Fig. 3. In addition, $\theta$ and the Galactic coordinates $(b, l)$ are related as $\cos \theta=$ $\cos b \cos l$.

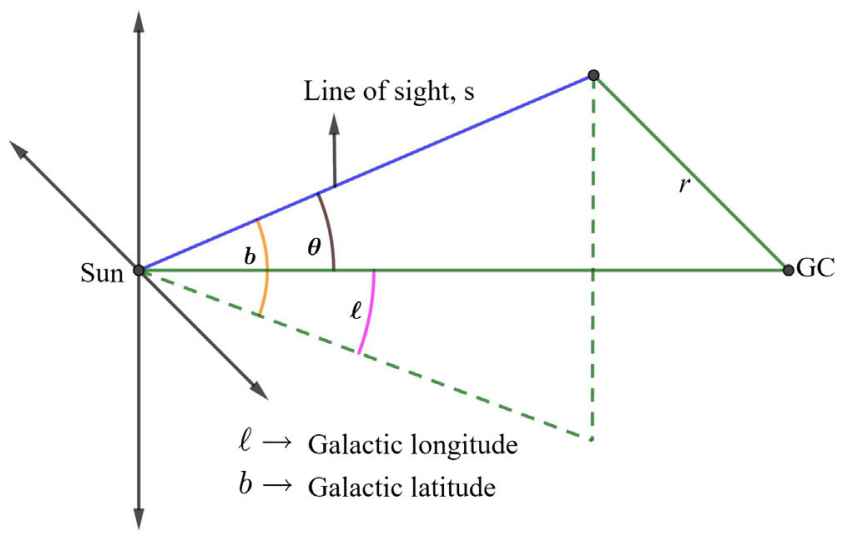

FIG. 3. The line of sight (LOS) $s$ for a gamma ray arriving at the Earth which originated at a point with distance $r$ from the Galactic Center (GC). 


\section{Gamma-ray spectrum from DM annihilation}

As mentioned already, the dominant DM annihilation channels in the triplet KNT model are those with the SM gauge bosons as final states. Hence, two types of gammaray spectra arise from SM gauge boson final states: (i) continuum spectra coming from $W^{+} W^{-}$and $Z Z$, and (ii) linelike spectra from $\gamma \gamma$ and $\gamma Z$ at $E_{\gamma}=M_{F_{1}}$ and $E_{\gamma}=M_{F_{1}}-\frac{M_{Z}^{2}}{4 M_{F_{1}}}$, respectively. Besides, the DM annihilation to $\gamma+X$ can also give linelike spectra at the end point of photon energy. The sensitivity studies for IACTs involving the continuum and linelike photon spectra require different strategies. In our study, we focus on the continuum spectra given by the $W^{+} W^{-}$final states as a representative channel. We have used PPPC4DMID [55] to calculate the gamma-ray spectra coming from the $W^{+} W^{-}$final states, which include the electroweak (EW) corrections [56] that become dominant at $O(\mathrm{TeV})$ energies. From Fig. 4, we can see the decrease in the gamma-ray spectra per annihilation with the mass of the DM.

In passing, let us make some remarks on the EW corrections implemented in the PPPC4DMID. At energies much higher than the electroweak scale, the dominant EW corrections for a typical $2 \rightarrow 2$ process arise from the Sudakov double logarithms, which are of the form $\delta_{\mathrm{DL}}^{(L)} \sim\left(\frac{\alpha}{4 \pi}\right)^{L} \log ^{2 L} \frac{s}{M_{W}^{2}}$ at $L$ th loop order [57-59]. Here, $\alpha$ is $\mathrm{SU}(2)$ coupling, and for the nonrelativistic DM, the centerof-mass energy is $s \sim 4 M_{\mathrm{DM}}^{2}$. As we can see from Fig. 5, the magnitudes of these EW corrections at one loop and two loop grow with the DM mass, and it indicates that for the DM mass close to or beyond $100 \mathrm{TeV}$, one needs to properly resum these Sudakov EW double logarithms and incorporate them into the PPPC4DMID. In addition, several studies have been undertaken [60-67] to precisely determine the indirect detection signatures in current and upcoming IACTs for both the linelike and continuum photon spectra coming from $\mathrm{TeV}$-scale or more massive DM annihilation. Besides, the improved estimates of the QCD uncertainties in Monte Carlo event generators might be relevant for the gamma-ray searches of the DM [68].

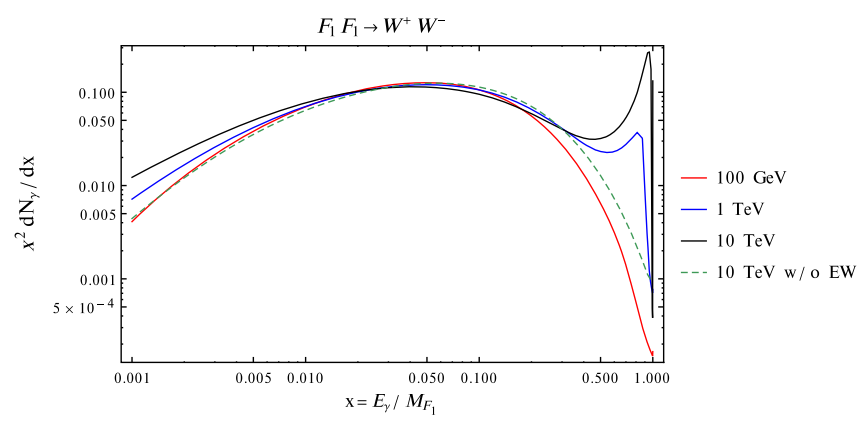

FIG. 4. Gamma-ray spectrum per annihilation determined using PPPC4DMID for the DM annihilation channel into $W^{+} W^{-}$.

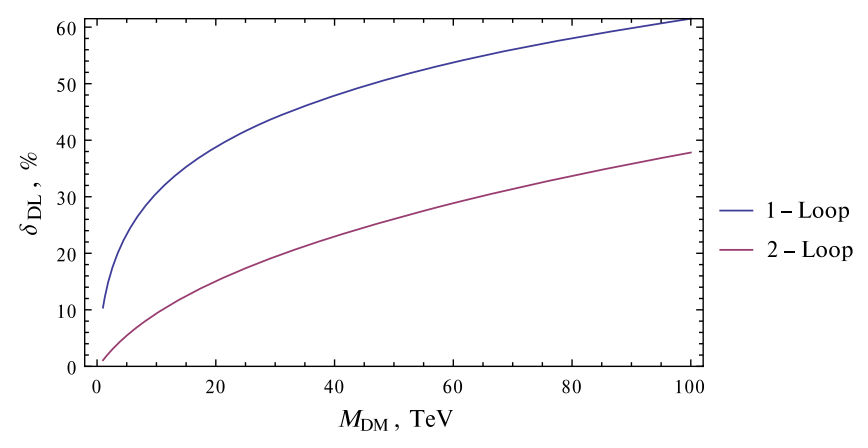

FIG. 5. The growing nature of the magnitude of the EW correction $\delta_{\mathrm{DL}}$ with the DM mass $M_{\mathrm{DM}}$ at one- and two-loop order necessitates the resummation of EW double logarithms.

\section{Astrophysical J factor}

The astrophysical $\mathrm{J}$ factor plays an essential role in determining the gamma-ray flux from DM annihilation, and therefore, one needs precise information of the DM density profile in the Milky Way, especially around the GC, which is the focus of this work. The $N$-body cosmological simulations are often used to parametrize the DM density profile of the Milky Way. The two most frequently used DM density profiles, that assume spherical symmetry, are the Navarro-Frenk-White (NFW) profile and the Einasto profile,

$$
\begin{gathered}
\rho_{\mathrm{NFW}}(r)=\rho_{s} \frac{r_{s}}{r}\left(1+\frac{r}{r_{s}}\right)^{-2}, \\
\rho_{\text {Ein }}(r)=\rho_{s} \exp \left\{-\frac{2}{\alpha}\left[\left(\frac{r}{r_{s}}\right)^{\alpha}-1\right]\right\},
\end{gathered}
$$

where $\rho_{s}$ and $r_{s}$ are the characteristic density and scale radius, respectively. The NFW profile behaves like $r^{-1}$ at the GC, whereas the Einasto profile does not and is smaller in magnitude. The $\alpha$ is the shape parameter that determines the steepness of the Einasto profile in the neighborhood of the GC. The numerical values of these parameters are $\left(\rho_{s}, r_{s}\right)=\left(0.184 \mathrm{GeV} \mathrm{cm}^{-3}, 24.42 \mathrm{kpc}\right)$ for the NFW profile, and $\left(\rho_{s}, r_{s}, \alpha\right)=\left(0.033 \mathrm{GeV} \mathrm{cm}^{-3}, 28.44 \mathrm{kpc}, 0.17\right)$ for the Einasto profile.

The value of the $\mathrm{J}$ factor is subject to considerable uncertainties, because there is no adequate observational data and precise simulation of our Galaxy with all its baryonic and DM content. Moreover, the DM density profile depends on the nature of the DM itself (for a review, please see Ref. [69]). Therefore, the J factors used in different studies vary significantly [70-72]. Hence, we have used the NFW profile, because of its cuspy nature at the GC, as the representative in our CTA sensitivity study to detect the DM of the KNT model. 


\section{DARK MATTER DETECTION WITH THE CTA}

\section{A. TeV DM at the CTA}

Gamma rays, being electrically neutral, do not deviate from their paths due to the Galactic magnetic field when propagating through the Galaxy. Moreover, gamma rays with energies ranging from $100 \mathrm{GeV}$ to $100 \mathrm{TeV}$ have minimal absorbance in the interstellar medium. So, when these very high-energy (VHE) gamma rays enter the Earth's atmosphere, they undergo collisions with atmospheric molecules and generate an air shower of secondary particles known as an extensive air shower. These shower particles descend to Earth with almost the speed of light, and due to the Cherenkov effect, these ultrarelativistic charged particles create a faint blue light in the air, which typically lasts for a few nanoseconds. Most of this Cherenkov light is emitted at altitudes ranging between 5 and $15 \mathrm{~km}$, and it propagates down to the ground level as a quasiplanar, thin disk of Cherenkov photons orthogonal to the shower axis. It may cover about $50000 \mathrm{~m}^{2}$ of an area on the ground. By placing arrays of IACTs within the projected Cherenkov light pool, it is possible to detect the air shower, provided that the mirror area of the telescope is large enough to catch enough photons. However, it is challenging to reconstruct the exact geometry of the air shower in space with the observation from a single telescope. Hence, multiple telescopes are deployed to take the image of a separate shower from different points, which leads to a stereoscopic reconstruction of the shower geometry. The images captured by IACTs after removing the background contributions show the track of the air shower, which points back to the celestial origin of the incident gamma rays, and eventually make it possible to determine the location of its source in the sky along with its spectral and spatial properties. As we have seen in Sec. III, when the DM of the triplet KNT model has a mass in the $O(\mathrm{TeV})$ range, its Sommerfeld enhanced annihilation into SM gauge bosons can produce the VHE gamma rays of either broad or narrow spectral features. Therefore, the IACTs are ideally suited to detect such TeV DM in the Galaxy.

There are three major currently operational IACTs: H.E.S.S., MAGIC, and VERITAS, which have performed well within their capabilities and have discovered many hundreds of VHE gamma-ray sources. However, the decisive point of the scientific performance of the CTA will be its ability to survey the sky over broad energy ranges from $20 \mathrm{GeV}$ to $300 \mathrm{TeV}$ with better angular resolution, energy reconstruction, and sensitivity, which is 1 order of magnitude better than existing IACTs. Besides this, to provide full sky coverage, the CTA will be installed at two different sites: one in the Northern Hemisphere at La Palma (Canary Islands, Spain) and another in the Southern Hemisphere at Paranal (Chile). The southern observatory (known as CTA South) will study the GC, and its northern counterpart (known as CTA North) will survey extragalactic objects. As the search for the DM at the GC will be a key focus of the CTA [27], we have chosen to study the detection possibility of the TeV DM of the triplet KNT model and to probe its parameter space.

\section{B. CTA instrument response function}

The instrument response function (IRF) can be considered as the area times the probability that a photon with a given set of input parameters is detected as an event with a set of observables (reconstructed parameters). For the CTA, the IRF is the product of effective area $\left(A_{\text {eff }}\right)$, the point spread function (PSF), energy resolution and dispersion, and the background rate as a function of energy. The key features of these quantities are as follows.

The effective area is the area within which the CTA can observe air showers. It depends on the energy of the primary gamma ray and the offset angle $\phi$ (the angle between the array's normal direction and the actual source position). Moreover, the point spread function (PSF) of CTA IRFs represents the spatial probability distribution of reconstructed event positions for a point source. Besides, in short, the energy dispersion of the CTA gives the resolution between the actual and reconstructed gamma-ray energies of events recorded by it. Finally, the background rate is the residual cosmic-ray background rate per solid angle (here, square degrees) as a function of reconstructed gamma-ray energy in the CTA.

As the CTA is not constructed yet, the CTA consortium has carried out several Monte Carlo simulations [73-75] to compute the results. We specifically make use of South $z 20 \_50 h$ in the CTA-Performance-prod3b-v2-FITS.tar.gz IRF (southern site with zenith angle $20^{\circ}$ and exposure time 50 hours) throughout our entire study. Let us point out a few factors for choosing this IRF. Due to its privileged location, the CTA southern site is most favorable for surveying the $\gamma$ sources in the GC with energy ranges from $20 \mathrm{GeV}$ to $300 \mathrm{TeV}$. Since our study is focused on the DM signal from the GC, we consider the IRFs designed for the southern site. Since it is assumed that the total operation time of CTA per year will be 1000 hours, we therefore expect that a longer exposure period is necessary to probe a possible a DM source region. From Fig. 6, we can see the variation of the effective area that will enter into our computation of gamma-ray counts due to DM or background in the CTA, with respect to gamma-ray energy and offset angle.

\section{CTA backgrounds}

In this section, we summarize the dominant background sources at the CTA considered for our study of detecting the DM signal from the KNT model.

(1) Cosmic rays: The cosmic rays (CRs) are highly energetic protons and nuclei. Apart from the VHE 

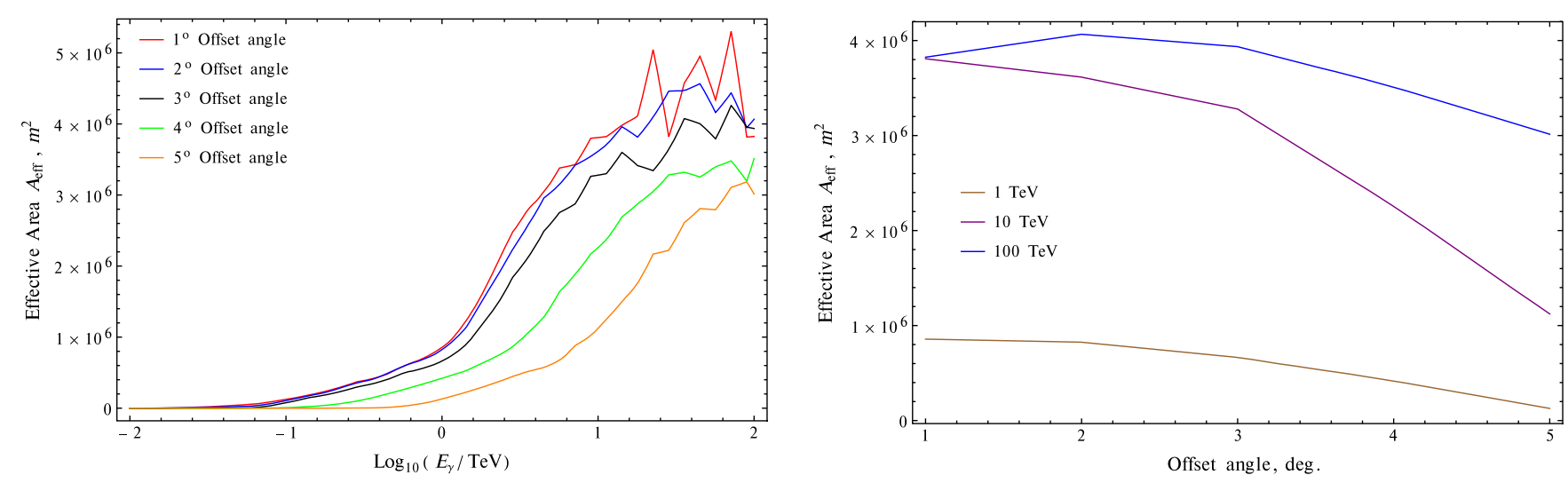

FIG. 6. Energy and offset angle dependency of the effective area of IRF for CTA southern site with zenith angle $20^{\circ}$ and exposure time 50 hours (S_z20_50h).

gamma rays, CRs also trigger air showers, which are $10^{3}$ times larger than those of $\gamma$ rays, and will act as the dominant background for the CTA. The CRs, during their flight from their origin, undergo deflection due to Galactic magnetic fields; only particles with sufficiently high energies can reach the Earth's atmosphere. While entering the Earth's atmosphere, CR protons undergo inelastic collisions with atmospheric air molecules, causing mixed hadronic and electromagnetic air showers. ${ }^{4}$ However, because of the large transverse momentum transfer due to hadronic interactions, the shower components are broad and exhibit irregular patterns compared to those of electromagnetic showers initiated by the primary gamma ray.

The shape of the captured image induced by the Cherenkov light from the air shower initiated by VHE gamma rays can be well approximated by an ellipse. But the image gathered from the CR-induced air shower has a distorted elliptical shape, which can allow one to discriminate between events generated due to gamma rays and those generated by cosmic rays. Nevertheless, a small fraction of images remain indistinguishable from the signal gamma rays, which constitute the irreducible background for the CTA.

(2) Galactic diffuse emission: Other than CR, Galactic diffuse emission (GDE) acts as a potential background for the CTA, which originates from the interaction of CR with interstellar molecules and atomic gas filling the Galactic plane. Observation of Fermi-LAT found that up to energy scales of $100 \mathrm{GeV}$, the GDE is dominated by $\pi^{0}$ decay, inverse Compton scattering, and bremsstrahlung radiation [78]. The contribution due to GDE

\footnotetext{
${ }^{4}$ There is a certain minimum threshold energy required in order to trigger the Cherenkov light shower by VHE- $\gamma$ and CRs. For electrons, muons, pions, and protons, the threshold energies are $21 \mathrm{MeV}, 3.4 \mathrm{GeV}, 5.6 \mathrm{GeV}$, and $38 \mathrm{GeV}$, respectively.
}

becomes significant when the region of interest becomes close to the GC, which is relevant for our case, since we are studying the possible DM sources near the GC.

Modeling TeV-range GDE for existing groundbased IACTs is a challenging task due to the uncertainties associated with the estimated background in the telescopes' field of view. It is also impossible to find a signal-free region in the sky by a priori consideration [76]. Besides, the data available from current experiments is limited in order to have a proper modeling of GDE at the $100 \mathrm{TeV}$ energy scale. As a consequence, we have taken a simplified approach following Refs. $[18,20,26]$ and left more realistic GDE modeling for a future work. We incorporate the GDE in our analysis using the P7V6 model of the Fermi team, ${ }^{5}$ which fits extremely well for the $50 \mathrm{MeV}$ and $500 \mathrm{GeV}$ energy range, and we use a power-law extrapolation of P7V6 data to $100 \mathrm{TeV}$. The H.E.S.S. observation of GDE from the Galactic ridge for Galactic coordinates $|b|<0.3^{\circ}$ and $|l|<0.8^{\circ}[77,79]$ is not taken into account, as it falls within the region that we have excluded from our study.

\section{Region of interest}

Our observation method is based on the Multi-RoI morphological analysis [20,26]. We assume that all telescopes are pointing toward the $\mathrm{GC}$ with Galactic longitude $\ell$ and latitude $b$ with coordinates $(0,0)$. The region of interest (RoI) is divided into five concentric circles spaced $1^{\circ}$ apart, such that the outermost circle has radius $5^{\circ}$, as seen in Fig. 7. The expected number of photon counts for signals (DM) and backgrounds (CR, GDE) in each circular RoI has been computed simultaneously. It is known that the central

\footnotetext{
${ }^{5}$ https://fermi.gsfc.nasa.gov/ssc/data/access/lat/Background Models.html.
} 

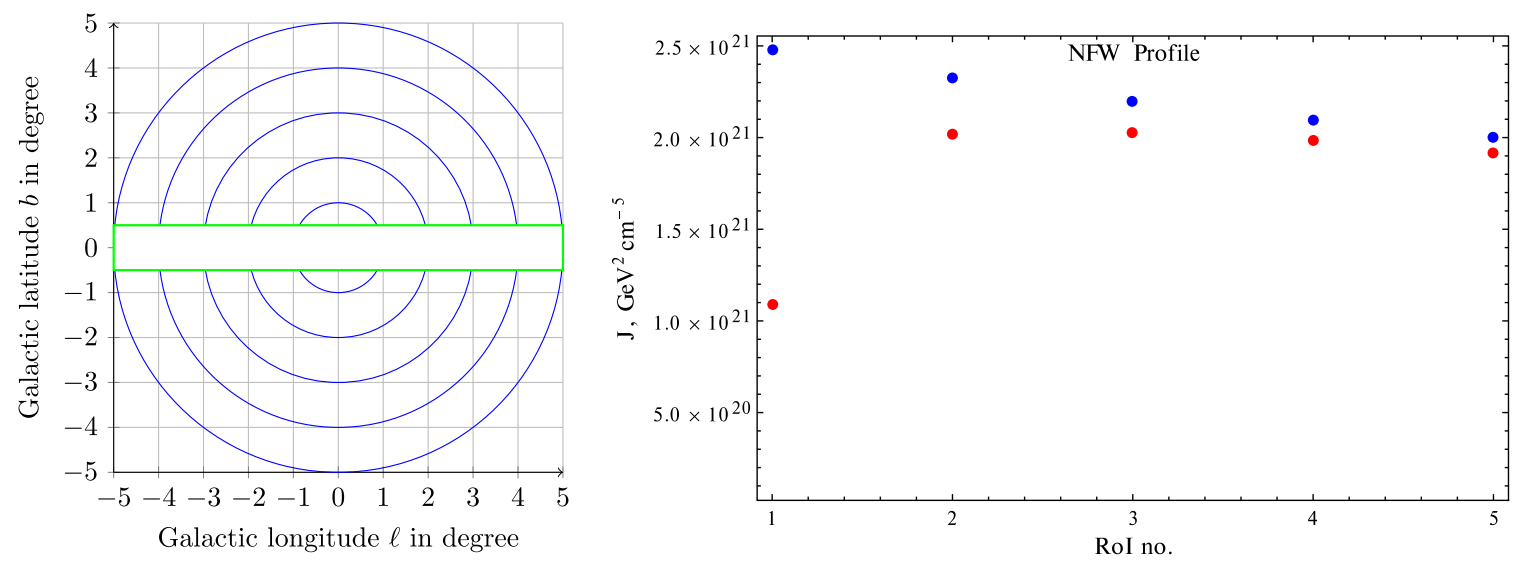

FIG. 7. Left: The annular region of interest (RoI), with circles of radius $1^{\circ}$ to $5^{\circ}$, and where the rectangular region $0.3^{\circ}<b<-0.3^{\circ}$ and $-5^{\circ}<\ell<5^{\circ}$ is excluded from each annular ring because of the large astrophysical background. Right: $\mathrm{J}$ factor corresponding to each annular region of interest determined for the NFW DM profile. Here, the blue points indicate the J-factor values for each annular ring. On the other hand, the red points are the J-factor values after subtracting the contribution from the central rectangular patch.

part of the GC is populated with several astrophysical sources, including Sagittarius $\mathrm{A}^{*}\left(\mathrm{SgrA}^{*}\right)$, emitting gamma-rays. In order to exclude photon counts coming from this region, we disregard the central part of the GC by introducing a rectangular patch with $0.3^{\circ}<b<-0.3^{\circ}$ and $-5^{\circ}<\ell<5^{\circ}$ within the RoI. The corresponding value of the $\mathrm{J}$ factor, considering the NFW profile, is shown in Fig. 7.

\section{ANALYSIS AND RESULTS}

\section{A. Expected gamma-ray counts at the CTA}

In this section, we obtain the expected counts for the dark matter, cosmic rays, and Galactic diffuse emission using the methods described below.

The expected differential count for each RoI $i$ and energy bin $j$ for the source $X$ is calculated using the relation

$$
\begin{aligned}
\frac{d \Gamma_{\gamma, i}^{X}}{d E_{\gamma}^{\prime}}= & \int_{\Omega_{i}^{\prime}} d \hat{p}^{\prime} \int d E_{\gamma} \int d \hat{p} \mathrm{~A}_{\mathrm{eff}}\left(E_{\gamma}, \hat{p}\right) \operatorname{PSF}\left(\hat{p}, \hat{p}^{\prime}\right) \\
& \times E_{\mathrm{disp}}\left(E_{\gamma}^{\prime}, E_{\gamma}, \hat{p}\right) \frac{d \phi_{\gamma}^{X}}{d E_{\gamma} d \Omega}\left(E_{\gamma}, \hat{p}\right),
\end{aligned}
$$

where the differential gamma-ray flux for the component $X$, $\frac{d \phi_{\gamma}^{X}}{d E_{\gamma} d \Omega}$, is weighted by the effective area $\mathrm{A}_{\mathrm{eff}}\left(E_{\gamma}, \hat{p}\right)$, point spread function $\operatorname{PSF}\left(\hat{p}, \hat{p}^{\prime}\right)$, and energy dispersion $E_{\text {disp }}\left(E_{\gamma}^{\prime}, E_{\gamma}, \hat{p}\right)$. Here, $\left(E_{\gamma}, \hat{p}\right)$ and $\left(E_{\gamma}^{\prime}, \hat{p}^{\prime}\right)$ denote the energy and direction of the actual and reconstructed gamma rays, respectively. The $\operatorname{PSF}\left(\hat{p}, \hat{p}^{\prime}\right)$, which is a probability distribution function of the angular separation between the actual and reconstructed gamma rays, is mostly relevant for a point source in the sky, but the sources we are considering - i.e. DM, CR, or GDE-are extended, so the PSF can be well approximated by a delta function. Moreover, the energy dispersion $E_{\text {disp }}$ is important if we are looking for a specific energy of the gamma ray, which applies to the linelike spectrum from DM annihilation. But, in our case, the resulting gamma-ray spectrum from DM annihilation has a continuum spectrum, so again weighting the differential gamma-ray flux with a Gaussian-like energy dispersion function is not important. Finally, the photon count is obtained using

$$
\mu_{i j}^{X}=T_{\mathrm{obs}} \int_{\Delta E_{j}} \mathrm{~d} E_{\gamma} \frac{\mathrm{d} \Gamma_{\gamma, i}^{X}}{\mathrm{~d} E_{\gamma}},
$$

where $T_{\text {obs }}$ is the total observation time, and the integration region $\Delta E_{j}$ denotes the $j$ th energy bin.

In our analysis, we divide our photon counts into 20 logarithmically spaced energy bins corresponding to the photon energy range, $E_{\gamma}=30 \mathrm{GeV}-M_{F_{1}}$, where the DM mass $M_{F_{1}}$ again varies from $1 \mathrm{TeV}$ to $100 \mathrm{TeV}$, using five RoIs as described in Sec. IV D. Also, the observation time is set to $T_{\text {obs }}=100 \mathrm{hr}$. For the DM annihilating into $W^{+} W^{-}$final states, the associated photon count is computed using Eqs. (21), (24), and (25) for the NFW profile.

Again, we compute the expected photon count for the GDE using Eqs. (24) and (25) from our simplified gammaray flux for the GDE, which is a power-law extrapolation up to $100 \mathrm{TeV}$ from the Fermi P7V6 model:

$$
\frac{\mathrm{d} \phi^{\mathrm{GDE}}}{\mathrm{d} E_{\gamma}}=1.0064 \times 10^{-6}\left(\frac{E_{\gamma}}{\mathrm{GeV}}\right)^{-2.333} \mathrm{GeV}^{-1} \mathrm{~cm}^{-2} \mathrm{~s}^{-1} \mathrm{sr}^{-1} .
$$

The expected gamma-ray count associated with CRs is evaluated using ctools version 1.6.2 [80], which is a software toolkit designed for data analysis of the CTA and other IACTs. The ctools consist of a set of binary executable $\mathrm{C}++$ and Python tools for performing the 


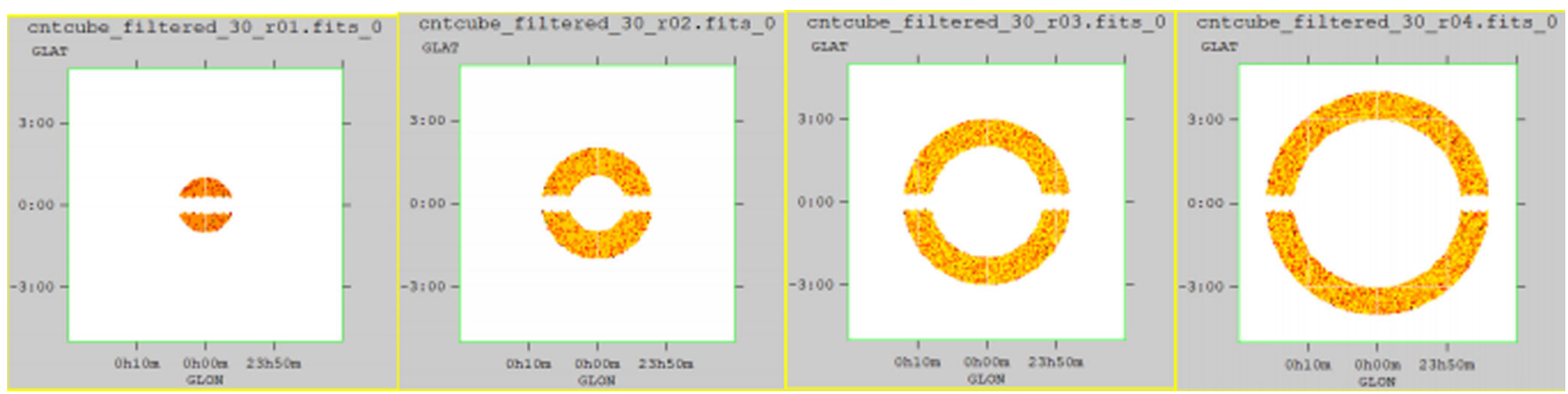

FIG. 8. Expected gamma-ray counts due to the CR background determined by the ctools from the first four RoIs, where the energy range is from $30 \mathrm{GeV}$ to $30 \mathrm{TeV}$.

necessary data analysis, where each of these tools needs a set of parameters like telescope pointing direction, radius of the field of view, pixelation, time period of observation, datebase calibration, etc. Equipped with IRF South_z20_50h, we use four components of the ctools in our analysis: ctobssim, ctbin, ctcubemask, and csresspec, following the sequence

ctobssim $\rightarrow$ ctbin $\rightarrow$ ctcubemask $\rightarrow$ csresspec.

Although csresspec inspects the spectral fit residual in ctools, we use it to extract the count per energy bin. Besides this, ctcubemask of ctools 1.6.2 does not have an option to carry out rectangular masking of the region $0.3^{\circ}<b<-0.3^{\circ}$ and $-5^{\circ}<\ell<5^{\circ}$. To perform this task, we exclude the region from longitude $-5^{\circ}$ to $5^{\circ}$ using overlapping circles of radius $0.3^{\circ}$. The output from ctools of the expected gamma-ray counts due to the cosmic rays from the first four RoIs are shown in Fig. 8.

In addition, as seen in Fig. 9, for both the first (innermost) and fifth (outermost) RoIs, the Sommerfeld enhancement in the DM annihilation allows its count rate to be comparable with the CR count. Moreover, we can see that

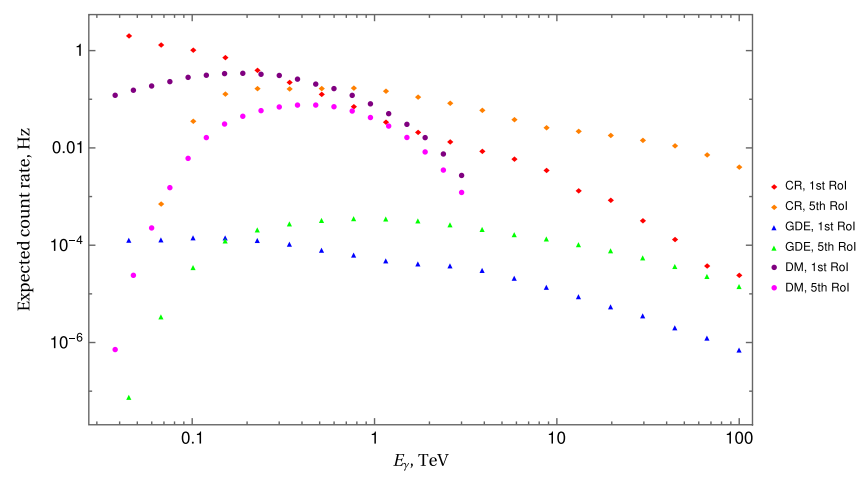

FIG. 9. Expected count rates from DM, CRs, and GDE with respect to the gamma-ray energy $E_{\gamma}$ at the CTA for the first and fifth RoIs. Here, the SE annihilation cross section $\langle\sigma v\rangle=$ $2.6 \times 10^{-23} \mathrm{~cm}^{3} \mathrm{~s}^{-1}$ for a DM mass $M_{F_{1}}=3 \mathrm{TeV}$ is used to calculate the expected count rate for the DM. in the case of CR and GDE, at low energy, the expected count rates are smaller in the fifth RoI than that in the first RoI. On the contrary, when the gamma-ray energy becomes higher, the count rate in the fifth RoI becomes larger compared to the first RoI. In Fig. 6, one can see that for low energy, the effective area of the CTA is small and increases with the gamma energy. Besides this, the incident gamma ray at a large angle with respect to the CTA telescope axis needs to have comparatively higher energy to initiate Cherenkov light that is detectable at the CTA. For this reason, we see a low count rate at low energy in the fifth RoI. On the other hand, with increasing angle towards the outer RoIs, the combination $\Omega_{i} \times A_{\text {eff }}$ becomes large at higher energies, and therefore shows higher gamma-ray counts at high energies for the CRs and GDE. In the case of $\mathrm{DM}$, the expected count rate is proportional to the $\mathrm{J}$ factor, which slightly decreases towards the outer RoI, as seen in Fig. 7. For that reason, we see that the expected count rate for DM is large to some extent in the first RoI compared to the fifth one.

The impact of the Sommerfeld enhancement on the DM annihilation is seen again in Fig. 10, where we consider the total expected count rate in each RoI for the DM, CRs, and

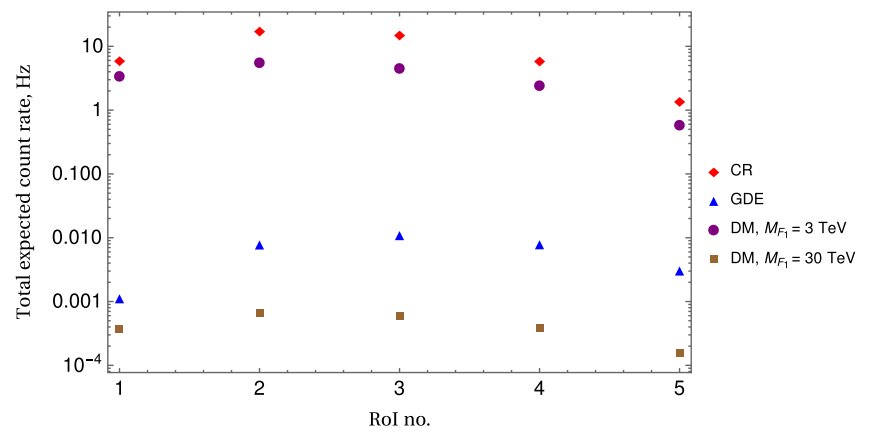

FIG. 10. Total expected count rates from DM, CRs, and GDE at each RoI. Here, the total expected count rate at the $i$ th RoI is given by $\frac{\sum_{j} \mu_{i j}}{T_{\mathrm{obs}}}$. Moreover, as the $\mathrm{SE}$ annihilation cross sections for DM masses $3 \mathrm{TeV}$ and $30 \mathrm{TeV}$ are $\sigma v=2.6 \times 10^{-23} \mathrm{~cm}^{3} \mathrm{~s}^{-1}$ and $\sigma v=1.3 \times 10^{-26} \mathrm{~cm}^{3} \mathrm{~s}^{-1}$, respectively, the total expected count rates for $3 \mathrm{TeV} \mathrm{DM}$ are comparable to CR counts. 
GDE. In this case, the variation of the expected count rate with respect to gamma-ray energy at each RoI is averaged out.

\section{B. Likelihood analysis}

In this section, we combine the expected gamma-ray counts coming from DM, CRs, and GDE backgrounds at the CTA, and determine its prospect to detect the DM in the triplet KNT model.

We divide our photon counts into 20 logarithmically spaced energy bins and five RoIs in a procedure similar to those in Refs. $[18,26]$ using the binned Poisson likelihood analysis. The counts are labeled with $\mu_{i j}^{X}$, where $X$ indicates the source-DM, CRs, or GDE-and the lower indices indicate the count in the $i$ th energy bin and the $j$ th RoI. The number of expected counts is then given by

$$
\mu_{i j}=\mu_{i j}^{\mathrm{DM}}+R_{i}^{\mathrm{CR}} \mu_{i j}^{\mathrm{CR}}+R_{i}^{\mathrm{GDE}} \mu_{i j}^{\mathrm{GDE}},
$$

where we have introduced the rescaling parameters $\left\{\mathbf{R}^{\mathrm{CR}}, \mathbf{R}^{\mathrm{GDE}}\right\}$. We may now write the likelihood function as the product of the independent Poisson distributions associated with each energy bin and RoI:

$$
L\left(\boldsymbol{\mu}, \boldsymbol{R}^{\mathrm{CR}}, \boldsymbol{R}^{\mathrm{GDE}} \mid \boldsymbol{n}\right)=\prod_{i j} \frac{\mu_{i j}^{n_{i j}}}{n_{i j} !} e^{-\mu_{i j}},
$$

where $n_{i j}$ represents the number of observed counts. However, we do not simulate individual Poisson realizations of the observed counts $n_{i j}$, but instead obtain an Asimov dataset [81]. In this procedure, the entire observed count is considered to be the sum of $\mathrm{CR}$ and GDE backgrounds only, and it can be found by setting $\mu_{i j}^{\mathrm{DM}} \rightarrow 0$ and $R_{i}^{\mathrm{CR} / \mathrm{GDE}} \rightarrow 1$ in Eq. (27).

Furthermore, we account for the systematic uncertainties in the signals by introducing a new set of Gaussian distributed nuisance parameters $\alpha_{i j}$, with mean 1 and standard deviation $\sigma_{\alpha}$. This approach results in a modification of Eq. (28) so that the new likelihood function is

$$
L(\boldsymbol{\mu}, \boldsymbol{\theta} \mid \boldsymbol{n})=\prod_{i j} \frac{\left(\alpha_{i j} \mu_{i j}\right)^{n_{i j}}}{\sqrt{2 \pi} \sigma_{\alpha} n_{i j} !} e^{-\alpha_{i j} \mu_{i j}} e^{-\frac{\left(\alpha_{i j}-1\right)^{2}}{2 \sigma_{\alpha}^{2}}},
$$

containing the nuisance parameters we denote collectively by the set $\boldsymbol{\theta}=\left\{\boldsymbol{\alpha}, \boldsymbol{R}^{\mathrm{CR}}, \boldsymbol{R}^{\mathrm{GDE}}\right\}$.

We separate our analysis into three distinct parts:

(1) First, we consider neglecting systematic uncertainties completely, as well as neglecting the photon count from GDE $\left(\boldsymbol{\alpha} \rightarrow 1, \sigma_{\alpha} \rightarrow 0, \mu_{i j}^{\mathrm{GDE}} \rightarrow 0\right)$.

(2) Next, we consider the effect of including GDE while still neglecting systematic uncertainties $\left(\boldsymbol{\alpha} \rightarrow 1, \sigma_{\alpha} \rightarrow 0\right)$.
(3) Finally, we consider systematic uncertainties restricted to $1 \%\left(\sigma_{\alpha} \rightarrow 0.01\right)$.

We now use maximum-likelihood estimates of the nuisance parameters separately in each of the above three cases to evaluate the test statistic

$$
\lambda\left(m_{F_{1}},\langle\sigma v\rangle\right)=-2 \log \left(\frac{L\left(\boldsymbol{\mu}, \boldsymbol{\theta}^{\prime}{ }_{\mathrm{MLE}} \mid \boldsymbol{n}\right)}{L\left(\boldsymbol{\mu}_{\mathrm{MLE}}, \boldsymbol{\theta}_{\mathrm{MLE}} \mid \boldsymbol{n}\right)}\right),
$$

where the subscript MLE indicates maximum-likelihood estimates of the underlying parameters, and we suppress the implicit dependence on the DM mass $m_{F_{1}}$ and cross section $\langle\sigma v\rangle$ in $\boldsymbol{\mu}$ for notational clarity. (Recall that $\mu_{i j}^{\mathrm{DM}} \sim\langle\sigma v\rangle$.) $\lambda$ is asymptotically equivalent to a $\chi^{2}$ distribution with one degree of freedom [since $\boldsymbol{\mu}$ in Eq. (30) is still a free parameter]. We bind the rescaling factors in Eq. (27) so that $0.5 \leq R_{i}^{\mathrm{CR}} \leq 1.5$ and $0.2 \leq R_{i}^{\mathrm{GDE}} \leq 5$ (in accordance with Ref. [18]), and we require $\boldsymbol{\alpha} \geq 0$ in order to keep probabilities non-negative in Eq. (29). While we formally maximize the likelihood function, in practice we minimize the negative log-likelihood function:

$$
\begin{aligned}
& -\log L(\boldsymbol{\mu}, \boldsymbol{\theta} \mid \boldsymbol{n}) \\
& =-\sum_{i j}\left(n_{i j} \log \left(\alpha_{i j} \mu_{i j}\right)-\alpha_{i j} \mu_{i j}-\frac{\left(\alpha_{i j}-1\right)^{2}}{2 \sigma_{\alpha}^{2}}\right),
\end{aligned}
$$

where we neglect constants and terms involving $n_{i j}$, which ultimately cancel in evaluating $\lambda$ and hence do not affect our analysis. Furthermore, we reduce the set of nuisance parameters by first analytically maximizing $\log L$ with respect to $\alpha_{i j}$ :

$$
\frac{\partial \log L}{\partial \alpha_{i j}}(\boldsymbol{\mu}, \boldsymbol{\theta} \mid \boldsymbol{n})=0,
$$

which, upon imposing the above mentioned constraint $\alpha_{i j} \geq 0$, gives

$\alpha_{i j}=\frac{1}{2}\left(1-\sigma_{i j}^{2} \mu_{i j}+\sqrt{1+4 \sigma_{\alpha}^{2} n_{i j}-2 \sigma_{\alpha}^{2} \mu_{i j}+\sigma_{\alpha}^{4} \mu_{i j}^{2}}\right)$,

which we substitute back into Eqs. (29) and (31). Note that $\alpha_{i j} \rightarrow 1$ in the limit $\sigma_{\alpha} \rightarrow 0$. With the explicit dependence on $\boldsymbol{\alpha}$ eliminated from the likelihood function in Eq. (29), we may regard the nuisance parameters as the reduced set $\boldsymbol{\theta}=\left\{\boldsymbol{R}^{\mathrm{CR}}, \boldsymbol{R}^{\mathrm{GDE}}\right\}$. At this stage, we wish to find an upper limit of the $95 \%$ confidence interval for $\langle\sigma v\rangle$. Towards this end, we numerically calculate $\lambda$ : first, we find the denominator in Eq. (30), thereby obtaining $\boldsymbol{\mu}_{\mathrm{MLE}}$; next, we modify the numerator by gradually increasing $\langle\sigma v\rangle$ until $\lambda$ reaches 2.71. [Recall that $\chi_{1}^{2} \sim Z^{2}$ with $Z \sim N(0,1)$, and hence for finding the upper limit of the $95 \%$ confidence interval, we 


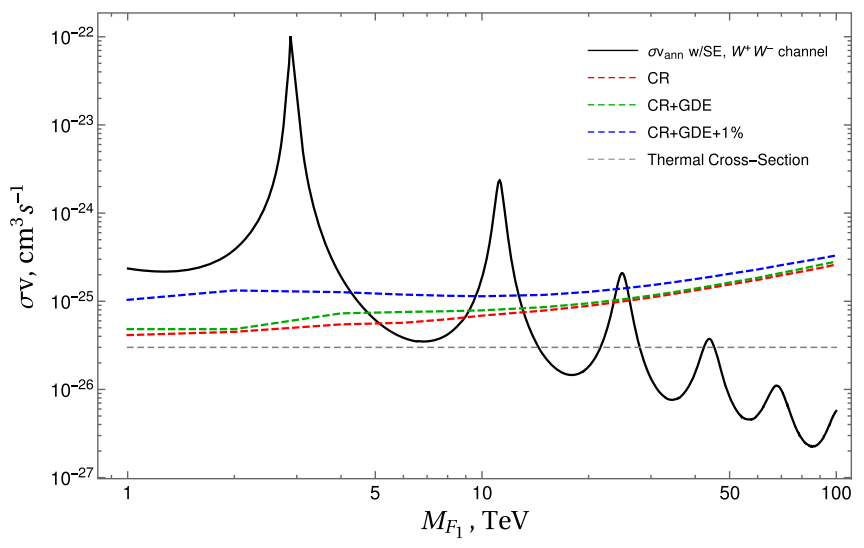

FIG. 11. The projected upper limit on the annihilation cross section for the triplet dark matter into the $W^{+} W^{-}$final state for the NFW profile and 100 hours of observation at the CTA.

require $Z^{2} \approx 1.645^{2} \approx 2.71$.] We subsequently use this value of $\langle\sigma v\rangle$ for the 95\% upper limit in the exclusion line for one particular fixed mass $m_{F_{1}}$. We then repeat this method for masses ranging from $1 \mathrm{TeV}$ to $100 \mathrm{TeV}$, thereby obtaining an exclusion line for $\langle\sigma v\rangle$. Finally, we repeat this entire process in the three distinct scenarios mentioned above-i.e., with the following backgrounds: CRs alone; CRs and GDE; as well as CRs, GDE, and 1\% systematic uncertainties. All of these results are illustrated in Fig. 11.

\section{Results and discussion}

In Fig. 11, we can see that the CTA can probe the DM of the triplet KNT model with the mass up to $25.7 \mathrm{TeV}$. The DM mass range $1.5 \mathrm{TeV} \leq M_{F_{1}} \leq 4.25 \mathrm{TeV}$, where the direct detection constraint sets the lower limit [Fig. 2 (right)], can be excluded from the CTA observation of continuum gamma rays coming from DM annihilation at the GC. However, the CTA sensitivity derived here is subjected to several issues, which we discuss below.

In our study, one prominent uncertainty which influences the expected gamma-ray count from the DM annihilation at the CTA and may degrade its DM detection sensitivity is the astrophysical $\mathbf{J}$ factor in Eq. (21). As the astrophysical $\mathbf{J}$ factor depends on the DM density profile of the Milky Way, the precise information of its density profile near the GC is essential for reducing the uncertainty that translates into the $\mathrm{J}$ factor used for calculating gamma-ray flux. We have used the NFW profile, which is cuspy at the GC, to calculate the corresponding $\mathbf{J}$ factors for our annular RoIs centered on the GC. However, one could use joint profile likelihood analysis, as was done, for example, in Ref. [66], to set upper limits on both the DM annihilation cross section and the J-factor values associated with the considered RoIs.

Also, the proper treatment of the background in RoIs contributes to the CTA sensitivity for DM detection. In our case, we consider the gamma rays from DM annihilation, and the background CRs and GDE simultaneously from the same region of the sky. One could have considered two regions of the sky, one where the count from the DM signal is expected to be high, known as the "ON" region, and an adjacent DM-signal-free but background-dominated region taken as "OFF." The statistical significance of the DM signal is determined in this scenario by contrasting the counts from the ON and OFF regions. However, in such an ON-OFF method, it is not always possible to define the signal-free region beforehand for extended sources like the DM, which could lead to systematic uncertainties in the counts and eventually incorrect substantial significance. The method we use is less prone to systematic uncertainties, as all the counts are taken for the same region.

Moreover, our extrapolation of the GDE up to $100 \mathrm{TeV}$ from the Fermi P7V6 model given by Eq. (26) is simplified, as the GDE can have nontrivial spatial and spectral properties at such high energies. For this reason, we have derived CTA sensitivity with and without the GDE. If we consider only the CRs as the background, we get the most stringent limit (the red dashed line in Fig. 11) on the DM annihilation cross section into $W^{+} W^{-}$final states in the triplet KNT model at the GC from our analysis. The inclusion of the GDE reduces the sensitivity, as seen from the green dashed line in Fig. 11. Finally, as expected, the systematics of $1 \%$ further degrades the CTA sensitivity, as illustrated by the blue dashed line in Fig. 11.

\section{CONCLUSION AND OUTLOOK}

In this work, we have studied the sensitivity of the upcoming Cherenkov Telescope Array to detect the DM candidate, with mass in the TeV range, of the KNT model annihilating into $W^{+} W^{-}$at the Galactic Center. In the following, we summarize our key results in order.

As shown in Sec. II, the KNT model can be generalized with large fermionic multiplets $\mathbf{F}_{i}$ of three generations and the scalar multiplet $\Phi$, all of them having the same integer isospin $j$ of $S U(2)_{L}$, and hypercharges $Y_{F_{i}}=0$ and $Y_{\Phi}=1$, respectively, without changing the topology of three-loop neutrino mass generation. Here, we set the upper limit on the value of the $S U(2)_{L}$ isospin for the KNT model using the bounds from the partial-wave unitarity on the coupled channels, $F_{i}^{(Q)} F^{(-Q)} \rightarrow W^{+} W^{-}, Z Z, \gamma \gamma, \gamma Z$. The bound on the $\mathrm{SU}(2)$ isospin of the fermion multiplet is $j_{F} \leq 8$ for one generation and $j_{F} \leq 7$ for three generations. The inclusion of the elastic channels in the coupled-channel analysis makes the partial-wave unitarity bound somewhat ineffective because of the Coulomb singularity arising from the photon exchange in $F_{i}^{(Q)} F_{i}^{(-Q)} \rightarrow F_{i}^{(Q)} F_{i}^{(-Q)}$.

Therefore, a more refined bound on the $S U(2)$ isospin comes from the appearance of the low-scale Landau pole in the gauge coupling in the presence of large electroweak multiplets. From Table I, we can see that the $j=3$ case is almost ruled out, and $j=2$ is already in tension with the nonobservation of nonperturbative $S U(2)$ coupling and the 
new physics appearing at the LHC. It leaves the minimal KNT with singlets and the triplet KNT with $j=1$ as the only viable models within the class of such three-loop radiative neutrino mass generation models.

However, the regions of parameter space associated with the dark matter of the singlet and triplet KNT models are different, because the DM of the singlet KNT does not have any SM gauge interaction, and its thermal freeze-out and annihilation at the present day are controlled by the KNT Yukawa couplings, which directly connects them to the neutrino mass generation and the related low-energy constraints. For this reason, the viability of the singlet KNT DM with $O(\mathrm{TeV})$ mass is left for a future study. On the other hand, the DM of the triplet KNT model, being charged under the SM gauge group, can have a large Sommerfeld enhancement, and its parameter space is determined mostly by the gauge interactions when the DM mass is in the TeV range.

Besides this, the SE annihilation of such heavy DM can produce detectable VHE gamma rays at the IACTs. We show that the DM of the triplet KNT model annihilating into $W^{+} W^{-}$at the $\mathrm{GC}$ can be probed up to $25.7 \mathrm{TeV}$ by the future CTA experiment considering the NFW profile and 100 hours of observation. However, there is some room for improvement of our sensitivity analysis. The triplet KNT model also has SE annihilation channels into $\gamma \gamma$ and $\gamma Z$, which would have a linelike signature in the gamma-ray spectrum around $E_{\gamma} \sim M_{F_{1}}$. Although the statistical analyses related to the continuum and linelike gamma-ray spectra have some differences, a proper combination of both analyses can lead to a better CTA sensitivity. Also, the consideration of the morphological analyses adopted in Refs. $[18,27,29]$ would result in an improved sensitivity of the CTA to detect the triplet KNT DM.

In addition, one can consider the dwarf spheroidal galaxies (dSphs) that are expected to contain up to $O\left(10^{3}\right)$ times more mass in DM than in visible matter and have a lower astrophysical gamma-ray background than the GC. Such characteristics of dSphs make them an ideal environment to search for the DM at the CTA [27]. Furthermore, the electrons produced from DM annihilation (mostly as secondary) can generate synchrotron radiation in the presence of the magnetic field, which could be observed as a diffuse radio emission in present and future radio observatories like the Square Kilometer Array (SKA) [84], and the sensitivity to detect such radio signals from DM in the dSphs is promising $[82,83]$. Therefore, a combined sensitivity study of the CTA and SKA to detect the DM in the $\mathrm{dSphs}$ will enable us to probe further the parameter space of the generalized KNT model [85] and other variants [86,87] of the three-loop radiative neutrino mass generation models.

\section{ACKNOWLEDGMENTS}

We would like to thank Casas Balázs, Adil Jueid, Jürgen Knödlseder, Luca Di Luzio, Ernest Ma, Soebur Razzaque, Nicholas L. Rodd, and Piero Ullio for stimulating discussions. T. A.C. and S. N. would like to thank The Abdus Salam International Centre for Theoretical Physics for hospitality and support where part of the work was done. The work of S. N This is supported by the United Arab Emirates University (UAEU) research fund, UPAR Grant No. 31S434. S. H. is supported by the Prime Minister Fellowship, Prime Minister's Office, Government of Bangladesh. This research has made use of the CTA instrument response functions provided by the CTA Consortium and Observatory; see http://www.ctaobservatory.org/science/cta-performance/ (version prod3b-v2) for more details. Also, this research made use of ctools, a community-developed analysis package for Imaging Air Cherenkov Telescope data. ctools is based on GammaLib, a community-developed toolbox for the scientific analysis of astronomical gamma-ray data.

\section{APPENDIX A: HELICITY AMPLITUDES AND PARTIAL-WAVE UNITARITY}

The helicity eigenstates of the fermion $\left(u_{ \pm}\right)$and antifermion $\left(v_{ \pm}\right)$with respect to the 3-momentum

$$
\vec{p}=(p \sin \theta \cos \phi, p \sin \theta \sin \phi, p \cos \theta)
$$

are given by

$$
\begin{gathered}
u_{+}(\vec{p})=\sqrt{E+m}\left(\cos \frac{\theta}{2}, e^{i \phi} \sin \frac{\theta}{2}, \frac{p}{E+m} \cos \frac{\theta}{2}, \frac{p}{E+m} e^{i \phi} \sin \frac{\theta}{2}\right), \\
u_{-}(\vec{p})=\sqrt{E+m}\left(-\sin \frac{\theta}{2}, e^{i \phi} \cos \frac{\theta}{2}, \frac{p}{E+m} \sin \frac{\theta}{2},-\frac{p}{E+m} e^{i \phi} \cos \frac{\theta}{2}\right), \\
v_{+}(\vec{p})=\sqrt{E+m}\left(\frac{p}{E+m} \sin \frac{\theta}{2},-\frac{p}{E+m} e^{i \phi} \cos \frac{\theta}{2},-\sin \frac{\theta}{2}, e^{i \phi} \cos \frac{\theta}{2}\right), \\
v_{-}(\vec{p})=\sqrt{E+m}\left(\frac{p}{E+m} \cos \frac{\theta}{2}, \frac{p}{E+m} e^{i \phi} \sin \frac{\theta}{2}, \cos \frac{\theta}{2}, e^{i \phi} \sin \frac{\theta}{2}\right),
\end{gathered}
$$


where \pm denotes either along or opposite to the given momentum direction. The incoming momenta of fermions and antifermions are taken along the $z$ axis $(\theta=0, \phi=0)$ and opposite to it $(\theta=\pi, \phi=\pi)$, respectively.

The transverse $(T)$ and longitudinal $(L)$ polarization 4-vectors of the outgoing gauge bosons with respect to the 3 -momentum $\vec{p}$ are given by

$$
\begin{aligned}
\epsilon_{T}(\hat{p}, \lambda)= & \frac{1}{\sqrt{2}}(0,-\lambda \cos \theta \cos \phi+i \sin \phi, \\
& -\lambda \cos \theta \sin \phi-i \cos \phi, \lambda \sin \theta), \quad \lambda= \pm 1,
\end{aligned}
$$

$\epsilon_{L}(\hat{p})=\frac{1}{M_{V}}(|\vec{p}|, E \sin \theta \cos \phi, E \sin \theta \sin \phi, E \cos \theta)$.

Therefore, if the outgoing gauge bosons are in the $x z$ plane, their corresponding polarization 4-vectors are given by setting $(\theta, \phi=0)$ and $(\pi-\theta, \phi=\pi)$, respectively.

In the high-energy limit, $\sqrt{s} \rightarrow \infty$, the helicity-conserving amplitude $\left(\left|\mu_{i}\right|=\left|\mu_{f}\right|\right)$ can be expanded as

$$
\mathcal{M}=\mathcal{M}_{\frac{1}{2}} s^{\frac{1}{2}}+O(1 / \sqrt{s})
$$

whereas the helicity-violating amplitude $\left(\left|\mu_{i}\right| \neq\left|\mu_{f}\right|\right)$ can be expanded as

$$
\mathcal{M}=\mathcal{M}_{1} s+\mathcal{M}_{0}+O(1 / s) .
$$

As we are taking into account the high-energy treelevel scattering, the above mentioned helicity amplitudes between the initial states, $|i\rangle=\left|F_{i}^{(Q)} F_{i}^{(-Q)}\right\rangle$, and final states, $|f\rangle=\left|V V^{\prime}\right\rangle$ (with $V V^{\prime}=W^{+} W^{-}, Z Z, \gamma \gamma, \gamma Z$ ), are considered to be the matrix elements $\mathcal{T}_{f i}$ of Eqs. (3) and (4). In the subsequent paragraphs, we avoid writing the values of subscripts $i$ and $f$ in $\mathcal{M}$ explicitly to have simplified notations.

We have checked that the cancellations among the $s$ channel, $t$ channel, and $u$ channel in $F F \rightarrow V V$ scattering render the terms $\mathcal{M}_{\frac{1}{2}}$ and $\mathcal{M}_{1}$ to be zero, which is expected, as $\mathbf{F}_{i}$ are the electroweak multiplets. In the following, we tabulate only the $\mathcal{M}_{0}$ terms of the corresponding helicity amplitudes.

For $F^{(Q)} F^{(-Q)} \rightarrow W^{+} W^{-}$, where $W^{ \pm}$are transverse, we have

$\mathcal{M}_{0}(+-;+-)=g^{2}\left[2 \tan \frac{\theta}{2}\left\{V_{-}\left(t_{3}\right) V_{+}\left(t_{3}-1\right)\right\}-t_{3} \sin \theta\right]$,

$$
\begin{aligned}
& \mathcal{M}_{0}(-+;+-) \\
& \quad=-g^{2}\left[2 \cot \frac{\theta}{2}\left\{V_{+}\left(t_{3}\right) V_{-}\left(t_{3}+1\right)\right\}+t_{3} \sin \theta\right],
\end{aligned}
$$

where the first two entries correspond to the fermion's helicity, $\pm \frac{1}{2}$, and the last two entries denote the gauge boson's helicity, \pm 1 . Also, $t_{3}$ is the eigenvalue of the diagonal generator, $T^{3}$, of $S U(2)$ associated with the component field, $F_{i}^{(Q)}$, of the multiplet, $\mathbf{F}_{i}$, and $t_{3}$ takes a value as $t_{3}=-j_{F},-j_{F}+1, \ldots, j_{F}-1, j_{F}$, if $j_{F}$ is the isospin of the fermionic multiplet. Besides, as the hypercharge of the fermion multiplet is zero, the electric charge of its component field follows $Q=t_{3}$. Moreover, $V_{ \pm}\left(t_{3}\right)=$ $\frac{1}{\sqrt{2}} \sqrt{\left(t \mp t_{3}\right)\left(t \pm t_{3}+1\right)}$ corresponds to $S U(2)$, raising and lowering factors with isospin $t$ and $t_{3}$.

In addition, for the scattering $F^{(Q)} F^{(-Q)} \rightarrow Z Z$, where $Z$ is transverse, we have

$$
\mathcal{M}_{0}(+-;+-)=\mathcal{M}_{0}(-+;-+)=\sqrt{2} g^{2} t_{3}^{2} \cos ^{2} \theta_{w} \tan \frac{\theta}{2},
$$

$\mathcal{M}_{0}(+-;-+)=\mathcal{M}_{0}(-+;+-)=-\sqrt{2} g^{2} t_{3}^{2} \cos ^{2} \theta_{w} \cot \frac{\theta}{2}$

Also, for $F^{(Q)} F^{(-Q)} \rightarrow \gamma \gamma$ scattering,

$\mathcal{M}_{0}(+-;+-)=\mathcal{M}_{0}(-+;-+)=\sqrt{2} g^{2} t_{3}^{2} \sin ^{2} \theta_{w} \tan \frac{\theta}{2}$,

$\mathcal{M}_{0}(+-;-+)=\mathcal{M}_{0}(-+;+-)=-\sqrt{2} g^{2} t_{3}^{2} \sin ^{2} \theta_{w} \cot \frac{\theta}{2}$.

Finally, we have, for $F^{(Q)} F^{(-Q)} \rightarrow \gamma Z$, where $Z$ is transverse,

$$
\mathcal{M}_{0}(+-;+-)=\mathcal{M}_{0}(-+;-+)=2 g^{2} t_{3}^{2} \sin \theta_{w} \cos \theta_{w} \tan \frac{\theta}{2},
$$

$\mathcal{M}_{0}(+-;-+)=\mathcal{M}_{0}(-+;+-)=-2 g^{2} t_{3}^{2} \sin \theta_{w} \cos \theta_{w} \cot \frac{\theta}{2}$.

Moreover, as we can see from Eqs. (A10)-(A17), the helicity amplitudes have initial total helicity either as $\mu_{i}=1$ or $\mu_{i}=-1$, and final total helicity either as $\mu_{f}=2$ or $\mu_{f}=-2$. Therefore the Wigner $d$ matrices of Eq. (4) will involve $d_{ \pm 1, \pm 2}^{J}$ with $J=2,3, \ldots$, corresponding to these helicity amplitudes. Since typically partial-wave amplitudes with higher $J$ values are smaller compared to those with lower possible $J$ values, we consider the partial-wave 
amplitudes with $J=2$ in our analysis. Hence, we have used the following Wigner $d$ matrices to calculate $a_{f i}^{J=2}$ :

$$
\begin{gathered}
d_{2,1}^{2}=-d_{1,2}^{2}=-\frac{1}{2}(1+\cos \theta) \sin \theta, \\
d_{2,-1}^{2}=-d_{-1,2}^{2}=-\frac{1}{2} \sin \theta(1-\cos \theta) .
\end{gathered}
$$

The coupled-channel matrix is written in the following basis:

$$
a^{J=2}=\left(\begin{array}{cccccc}
0 & 0 & 0 & 0 & \frac{g^{2}}{24 \sqrt{2} \pi} & \frac{g^{2}}{48 \pi} \\
0 & 0 & 0 & 0 & 0 & \frac{g^{2} \cos ^{2} \theta_{w}}{24 \sqrt{2} \pi} \\
0 & 0 & 0 & 0 & 0 & \frac{g^{2} \sin ^{2} \theta_{w}}{24 \sqrt{2} \pi} \\
0 & 0 & 0 & 0 & 0 & \frac{g^{2} \cos \theta_{w} \sin \theta_{w}}{24 \pi} \\
\frac{g^{2}}{24 \sqrt{2} \pi} & 0 & 0 & 0 & 0 & 0 \\
\frac{g^{2}}{48 \pi} & \frac{g^{2} \cos ^{2} \theta_{w}}{24 \sqrt{2} \pi} & \frac{g^{2} \sin ^{2} \theta_{w}}{24 \sqrt{2} \pi} & \frac{g^{2} \cos \theta_{w} \sin \theta_{w}}{24 \pi} & 0 & 0
\end{array}\right) .
$$

As we are only considering $F F \rightarrow V V^{\prime}$ scattering, the matrix elements of Eq. (A19) are zero for $V V^{\prime} \rightarrow V V^{\prime}$ and $F F \rightarrow F F$ channels. As the order of the coupled-channel matrix increases when the isospin of the fermion multiplet $j_{F}$ and the generation number $i$ increase, we first determine the largest eigenvalue of the corresponding coupled-channel matrix and identify the largest values of $j_{F}$ which satisfy the bound in Eq. (9) for single and three generations.

In the case of high-energy scattering of $F^{(Q)} F^{(-Q)} \rightarrow$ $F^{(Q)} F^{(-Q)}$ with $Q \neq 0$, the photon exchange at the $t$ channel would lead to the following helicity amplitudes:

$$
\begin{aligned}
\mathcal{M}_{0}^{(t)}\left(+\frac{1}{2},+\frac{1}{2} ;+\frac{1}{2},+\frac{1}{2}\right)= & 2 Q^{2} e^{2} \operatorname{cosec}^{2}(\theta / 2), \\
& \left(\mu_{i}=0, \mu_{f}=0\right),
\end{aligned}
$$

$$
\begin{array}{r}
\mathcal{M}_{0}^{(t)}\left(+\frac{1}{2},-\frac{1}{2} ;+\frac{1}{2},-\frac{1}{2}\right)= \\
2 Q^{2} e^{2} \cot ^{2}(\theta / 2), \\
\left(\mu_{i}=1, \mu_{f}=1\right) .
\end{array}
$$

From Eqs. (A20) and (A21), we can see that only Wigner $d$ matrices $d_{00}^{J}(\theta)$ and $d_{ \pm 1, \pm 1}^{J}(\theta)$ will be present in Eq. (4), but then the integration over $\cos \theta$ will be divergent because of the integrand's singularities at $\cos \theta= \pm 1$. This is the Coloumb singularity appearing in the elastic channels, which is mentioned in Sec. II A.

$$
\begin{aligned}
\left|\psi_{s c}\right\rangle= & \left(W^{+} W^{-}, Z Z, \gamma \gamma, \gamma Z, F_{i}^{0} F_{i}^{0}, \ldots,\right. \\
& \left.F_{i}^{(Q)} F_{i}^{(-Q)}, \ldots, F_{i}^{\left(j_{F}\right)} F_{i}^{\left(-j_{F}\right)}\right)^{T},
\end{aligned}
$$

where $i=1,2,3$ is the generation index, and for more than one generation of multiplets, the two-particle states $F_{i}^{(Q)} F_{i}^{(-Q)}$ are repeated for each value of $i$ in the above basis. For example, for isospin $j_{F}=1$ and one generation, it is given by

\section{APPENDIX B: LANDAU POLE}

The one-loop beta function for the $S U(2)$ coupling given in Eq. (11) is

$$
\begin{aligned}
\beta_{g} & =\frac{g^{3}}{16 \pi^{2}}\left(-\frac{19}{6}+\frac{4}{3} \sum_{i} \kappa_{F_{i}} T\left(F_{i}\right)+\frac{1}{3} \sum_{j} \eta_{S_{j}} T\left(S_{j}\right)\right) \\
& =\frac{g^{3}}{16 \pi^{2}} b_{g},
\end{aligned}
$$

where $b_{g}$ is denoting the factor of $g^{3} / 16 \pi^{2}$ in Eq. (B1). Therefore, the coupling $g(\mu)$ at the energy scale $\mu$ is given as

$$
g(\mu)=\frac{g\left(\mu_{0}\right)}{1-\frac{b_{g} g\left(\mu_{0}\right)^{2}}{8 \pi^{2}} \ln \left(\frac{\mu}{\mu_{0}}\right)},
$$

where $\mu_{0}$ is the reference energy scale and $g\left(\mu_{0}\right)$ is the value of coupling at that scale. Moreover, we denote $b_{g}^{\mathrm{SM}}=-19 / 6$ for the SM contribution only. Now, if $b_{g}$ is positive, we have an energy scale called the Landau pole, $\mu=\Lambda_{\mathrm{Lan}}>\mu_{0}$, for which the denominator of Eq. (B2) vanishes [provided $g\left(\mu_{0}\right) \neq 0$ ], and we get

$$
\Lambda_{\text {Lan }}=\mu_{0} \exp \left(\frac{8 \pi^{2}}{b_{g} g\left(\mu_{0}\right)^{2}}\right) .
$$

Now, for the generalized KNT model, there are three generations of $S U(2)$ fermion multiplets with isospin $j$ (where $j$ is integer) and hypercharge $Y=0$, and one $S U(2)$ 
scalar multiplet with isospin $j$ and hypercharge $Y=1$. Therefore, the factor $b_{g}$ is given by $b_{g}=-\frac{19}{6}+\frac{13}{9} j(j+1)$ $(2 j+1)$. Consequently, the factor $b_{g}$ becomes positive for isospin $j \geq 1$. We consider the electroweak (EW) scale at $\mu_{\mathrm{EW}}=M_{Z}=91.1876 \mathrm{GeV}$ with $g\left(M_{Z}\right)=0.65114$ $[48,90]$. If the masses of the additional KNT multipletswhich are, for simplicity, denoted by a common mass parameter, $M_{X}$ - are comparable to the electroweak scale, then the running of the $S U(2)$ coupling from the electroweak scale, $\mu_{0}=M_{Z}$, to the higher energy will involve positive $b_{g}$ for isospin $j \geq 1$, and correspondingly we determine the Landau pole of $g$ using Eq. (B3), which is tabulated in the second column of Table I for $j=1,2,3$.
Now, if the KNT mass scale is $M_{X} \gg M_{Z}$-i.e., in the TeV range- the contributions from the KNT fields to the running of the $S U(2)$ from $M_{Z}$ to $M_{X}$ can be considered negligible. In this case, we first determine $g\left(\mu=M_{X}\right)$ using Eq. (B1), with $b_{g}=b_{g}^{\mathrm{SM}}$ from the EW scale $\mu_{0}=M_{Z}$, that is up to the energy scale $M_{X}$, where the KNT fields' contributions in the running become relevant. Afterwards, we compute the Landau pole for isospin $j$ using Eq. (B3), with $\mu_{0}=M_{X}$ and $g\left(\mu_{0}=M_{X}\right)$ as inputs. We tabulate the corresponding Landau poles for the isospin $j=1,2,3$ in the third, fourth, and fifth columns of Table I for $M_{X}=10^{3}, 10^{4}$, and $10^{5} \mathrm{GeV}$, respectively.
[1] F. M. Rieger, E. de Ona-Wilhelmi, and F. A. Aharonian, arXiv:1302.5603.

[2] A. De Angelis and M. Mallamaci, Eur. Phys. J. Plus 133, 324 (2018).

[3] G. Di Sciascio, J. Phys. Conf. Ser. 1263, 012003 (2019).

[4] L. Bergstrom, P. Ullio, and J. H. Buckley, Astropart. Phys. 9, 137 (1998).

[5] T. Bringmann and C. Weniger, Phys. Dark Universe 1, 194 (2012).

[6] L. Rinchiuso et al. (H.E.S.S. Collaboration), EPJ Web Conf. 209, 01023 (2019).

[7] H. Abdallah et al. (H.E.S.S. Collaboration), Phys. Rev. Lett. 117, 111301 (2016).

[8] H. Abdallah et al. (H.E.S.S. Collaboration), Phys. Rev. Lett. 120, 201101 (2018).

[9] A. Abramowski et al. (H.E.S.S. Collaboration), Phys. Rev. Lett. 110, 041301 (2013).

[10] H. Abdalla et al. (H.E.S.S. Collaboration), Phys. Rev. Lett. 117, 151302 (2016).

[11] J. M. Paredes (MAGIC Collaboration), arXiv:2003.03841.

[12] M. Doro (MAGIC Collaboration), arXiv:1701.05702.

[13] B. Zitzer (VERITAS Collaboration), Proc. Sci., ICRC2017 (2018) 904.

[14] M. Actis et al., Exp. Astron. 32, 193 (2011).

[15] M. Doro et al. (CTA Consortium Collaboration), Astropart. Phys. 43, 189 (2013).

[16] B.S. Acharya et al. (CTA Consortium Collaboration), Astropart. Phys. 43, 3 (2013).

[17] M. Pierre, J. M. Siegal-Gaskins, and P. Scott, J. Cosmol. Astropart. Phys. 06 (2014) 024.

[18] H. Silverwood, C. Weniger, P. Scott, and G. Bertone, J. Cosmol. Astropart. Phys. 03 (2015) 055.

[19] L. Roszkowski, E. M. Sessolo, and A. J. Williams, J. High Energy Phys. 02 (2015) 014.

[20] V. Lefranc, E. Moulin, P. Panci, and J. Silk, Phys. Rev. D 91, 122003 (2015).

[21] A. Ibarra, A. S. Lamperstorfer, S. López-Gehler, M. Pato, and G. Bertone, J. Cosmol. Astropart. Phys. 09 (2015) 048.
[22] V. Lefranc, G. A. Mamon, and P. Panci, J. Cosmol. Astropart. Phys. 09 (2016) 021.

[23] V. Lefranc, E. Moulin, P. Panci, F. Sala, and J. Silk, J. Cosmol. Astropart. Phys. 09 (2016) 043.

[24] C. Bigongiari (CTA Consortium Collaboration), Nucl. Part. Phys. Proc. 279-281, 174 (2016).

[25] J. Conrad, AIP Conf. Proc. 1792, 030002 (2017).

[26] C. Balázs, J. Conrad, B. Farmer, T. Jacques, T. Li, M. Meyer, F. S. Queiroz, and M. A. Sánchez-Conde, Phys. Rev. D 96, 083002 (2017).

[27] B.S. Acharya et al. (CTA Consortium Collaboration), arXiv:1709.07997.

[28] A. Hryczuk, K. Jodlowski, E. Moulin, L. Rinchiuso, L. Roszkowski, E. M. Sessolo, and S. Trojanowski, J. High Energy Phys. 10 (2019) 043.

[29] A. Acharyya et al. (CTA Collaboration), arXiv:2007.16129.

[30] L. Rinchiuso, O. Macias, E. Moulin, N. L. Rodd, and T. R. Slatyer, Phys. Rev. D 103, 023011 (2021).

[31] L. M. Krauss, S. Nasri, and M. Trodden, Phys. Rev. D 67, 085002 (2003).

[32] Y. Cai, J. Herrero-García, M. A. Schmidt, A. Vicente, and R. R. Volkas, Front. Phys. 5, 63 (2017).

[33] C. S. Chen, K. L. McDonald, and S. Nasri, Phys. Lett. B 734, 388 (2014).

[34] A. Ahriche, C. S. Chen, K. L. McDonald, and S. Nasri, Phys. Rev. D 90, 015024 (2014).

[35] A. Ahriche, K. L. McDonald, and S. Nasri, J. High Energy Phys. 10 (2014) 167.

[36] A. Ahriche, K. L. McDonald, S. Nasri, and T. Toma, Phys. Lett. B 746, 430 (2015).

[37] T. A. Chowdhury and S. Nasri, Phys. Lett. B 782, 215 (2018).

[38] A. Ahriche and S. Nasri, J. Cosmol. Astropart. Phys. 07 (2013) 035.

[39] M. Cirelli, N. Fornengo, and A. Strumia, Nucl. Phys. B753, 178 (2006).

[40] M. Ibe, S. Matsumoto, and R. Sato, Phys. Lett. B 721, 252 (2013).

[41] J. McKay and P. Scott, Phys. Rev. D 97, 055049 (2018). 
[42] T. A. Chowdhury and S. Nasri, Phys. Rev. D 97, 075042 (2018).

[43] K. S. Babu, J. Julio, and Y. Zhang, Nucl. Phys. B858, 468 (2012).

[44] K. Hally, H. E. Logan, and T. Pilkington, Phys. Rev. D 85, 095017 (2012).

[45] M. Jacob and G. C. Wick, Ann. Phys. (N.Y.) 7, 404 (1959).

[46] A. Schuessler and D. Zeppenfeld, arXiv:0710.5175.

[47] L. Di Luzio, J. F. Kamenik, and M. Nardecchia, Eur. Phys. J. C 77, 30 (2017).

[48] L. Di Luzio, R. Gröber, J. F. Kamenik, and M. Nardecchia, J. High Energy Phys. 07 (2015) 074.

[49] L. Di Luzio, R. Gröber, and G. Panico, J. High Energy Phys. 01 (2019) 011.

[50] M. Beneke, C. Hellmann, and P. Ruiz-Femenia, J. High Energy Phys. 05 (2015) 115.

[51] I. Esteban, M. C. Gonzalez-Garcia, A. HernandezCabezudo, M. Maltoni, and T. Schwetz, J. High Energy Phys. 01 (2019) 106.

[52] N. Aghanim et al. (Planck Collaboration), Astron. Astrophys. 641, A6 (2020).

[53] E. Aprile et al. (XENON Collaboration), Phys. Rev. Lett. 121, 111302 (2018).

[54] J. Giedt, A. W. Thomas, and R. D. Young, Phys. Rev. Lett. 103, 201802 (2009).

[55] M. Cirelli, G. Corcella, A. Hektor, G. Hutsi, M. Kadastik, P. Panci, M. Raidal, F. Sala, and A. Strumia, J. Cosmol. Astropart. Phys. 03 (2011) 051.

[56] P. Ciafaloni, D. Comelli, A. Riotto, F. Sala, A. Strumia, and A. Urbano, J. Cosmol. Astropart. Phys. 03 (2011) 019.

[57] A. Denner and S. Pozzorini, Eur. Phys. J. C 18, 461 (2001).

[58] A. Denner, M. Melles, and S. Pozzorini, Nucl. Phys. B662, 299 (2003).

[59] A. V. Manohar and W. J. Waalewijn, J. High Energy Phys. 08 (2018) 137.

[60] M. Baumgart, I. Z. Rothstein, and V. Vaidya, Phys. Rev. Lett. 114, 211301 (2015).

[61] G. Ovanesyan, T. R. Slatyer, and I. W. Stewart, Phys. Rev. Lett. 114, 211302 (2015).

[62] M. Baumgart, I. Z. Rothstein, and V. Vaidya, J. High Energy Phys. 04 (2015) 106.

[63] G. Ovanesyan, N. L. Rodd, T. R. Slatyer, and I. W. Stewart, Phys. Rev. D 95, 055001 (2017).

[64] M. Baumgart, T. Cohen, I. Moult, N. L. Rodd, T. R. Slatyer, M. P. Solon, I. W. Stewart, and V. Vaidya, J. High Energy Phys. 03 (2018) 117.

[65] M. Baumgart, T. Cohen, E. Moulin, I. Moult, L. Rinchiuso, N. L. Rodd, T. R. Slatyer, I. W. Stewart, and V. Vaidya, J. High Energy Phys. 01 (2019) 036.
[66] L. Rinchiuso, N. L. Rodd, I. Moult, E. Moulin, M. Baumgart, T. Cohen, T. R. Slatyer, I. W. Stewart, and V. Vaidya, Phys. Rev. D 98, 123014 (2018).

[67] C. W. Bauer, N. L. Rodd, and B. R. Webber, arXiv:2007 .15001 .

[68] S. Amoroso, S. Caron, A. Jueid, R. Ruiz de Austri, and P. Skands, J. Cosmol. Astropart. Phys. 05 (2019) 007.

[69] P. Salucci, Astron. Astrophys. Rev. 27, 2 (2019).

[70] M. Pato, F. Iocco, and G. Bertone, J. Cosmol. Astropart. Phys. 12 (2015) 001.

[71] D. Hooper, Phys. Dark Universe 15, 53 (2017).

[72] M. Benito, A. Cuoco, and F. Iocco, J. Cosmol. Astropart. Phys. 03 (2019) 033.

[73] K. Bernlöhr, A. Barnacka, Y. Becherini et al., Astropart. Phys. 43, 171 (2013).

[74] T. Hassan, L. Arrabito, K. Bernlöhr et al., Astropart. Phys. 93, 76 (2017).

[75] A. Acharyya, I. Agudo, E. O. Angüner et al., Astropart. Phys. 111, 35 (2019).

[76] A. Neronov and D. Semikoz, Astron. Astrophys. 633, A94 (2020).

[77] F. Aharonian et al. (H.E.S.S. Collaboration), Nature (London) 439, 695 (2006).

[78] M. Ackermann, M. Ajello, and W. B. Atwood et al., Astrophys. J. 750, 3 (2012).

[79] A. Abramowski et al. (H.E.S.S. Collaboration), Phys. Rev. D 90, 122007 (2014).

[80] J. Knödlseder, M. Mayer, C. Deil, J. B. Cayrou, E. Owen, N. Kelley-Hoskins, C. C. Lu, R. Buehler, F. Forest, T. Louge, H. Siejkowski, K. Kosack, L. Gerard, A. Schulz, P. Martin, D. Sanchez, S. Ohm, T. Hassan, and S. Brau-Nogué, Astron. Astrophys. 593, A1 (2016).

[81] G. Cowan, K. Cranmer, E. Gross, and O. Vitells, Eur. Phys. J. C 71, 1554 (2011).

[82] S. Colafrancesco, M. Regis, P. Marchegiani, G. Beck, R. Beck, H. Zechlin, A. Lobanov, and D. Horns, Proc. Sci., AASKA14 (2015) 100.

[83] G. Beck, Galaxies 7, 16 (2019).

[84] A. Weltman, P. Bull, S. Camera et al., Pub. Astron. Soc. Aust. 37, e002 (2020).

[85] T. Chowdhury and S. Nasri (to be published).

[86] R. Cepedello, M. Hirsch, P. Rocha-Morán, and A. Vicente, J. High Energy Phys. 08 (2020) 067.

[87] M. Gustafsson, J. M. No, and M. A. Rivera, J. High Energy Phys. 11 (2020) 070.

[88] D. J. Gross and F. Wilczek, Phys. Rev. Lett. 30, 1343 (1973).

[89] F. Wilczek and A. Zee, Phys. Rev. D 25, 553 (1982).

[90] M. Tanabashi et al. (Particle Data Group), Phys. Rev. D 98, 030001 (2018). 